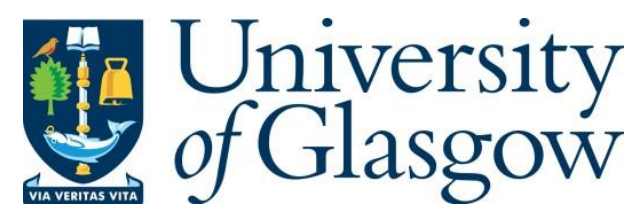

Sufian, A., Artigaut, M., Shire, T. and O'Sullivan, C. (2021) Influence of fabric on stress distribution in gap-graded soil. Journal of Geotechnical and Geoenvironmental Engineering, (doi: 10.1061/(ASCE)GT.1943-5606.0002487).

There may be differences between this version and the published version. You are advised to consult the publisher's version if you wish to cite from it.

http://eprints.gla.ac.uk/226312/

Deposited on: 20 November 2020

Enlighten - Research publications by members of the University of Glasgow http://eprints.gla.ac.uk 


\title{
Influence of Fabric on Stress Distribution in Gap-Graded Soil
}

\author{
Adnan Sufian*1,2, Marion Artigaut ${ }^{2}$, Thomas Shire ${ }^{3}$, and Catherine \\ O'Sullivan ${ }^{2}$ \\ ${ }^{1}$ School of Civil Engineering, The University of Queensland, Brisbane, Australia \\ ${ }^{2}$ Department of Civil and Environmental Engineering, Imperial College London, London, United \\ Kingdom \\ ${ }^{3}$ School of Engineering, University of Glasgow, Glasgow, United Kingdom
}

November 20, 2020

\begin{abstract}
The combined influence of density and stress-induced fabric anisotropy on the nature of stress transmission in gap-graded soils with cohesionless fines has been explored using the discrete element method (DEM). Various particle size ratios and fines contents were considered in simulations of constant mean stress triaxial compression. Analysis of the available particle-scale data focused on understanding how stress was distributed amongst and between the finer and coarser particles. While the study confirms that stress is transferred from the coarser to the finer fraction with increasing fines content, the concept of a threshold fines content at which there is a definitive transition in the nature of stress transmission is not supported. Rather there is a gradual evolution of the distribution of stresses between the two size fractions with increasing fines content, and the relationship between fines content and stress in the finer fraction depends on the size ratio, density and fabric anisotropy. For the denser samples considered, the stress transmitted by the finer fraction systematically reduced during shearing. An alternate definition of granular void ratio is introduced which accounts for the non-active fine and coarse particles and is formulated in a consistent manner to capture both the inter-granular and inter-fine void ratios commonly found in the literature, along with the equivalent granular void ratio. The anisotropy of the network of contacts formed by the interactions of coarse particles was observed to be the dominant contributor to fabric anisotropy.
\end{abstract}

${ }^{*}$ Corresponding author, email: a.sufian@uq.edu.au, ORCid: 0000-0003-2816-3250 


\section{Introduction}

Granular gap-graded soils comprise mixtures of coarser particles and cohesionless finer particles with no intermediate particle sizes. Understanding the behaviour of such gap-graded soils is relevant to phenomena including liquefaction (Lade and Yamamuro, 1997; Thevanayagam et al., 2002) and suffusion (Wan and Fell, 2008; Sail et al., 2011). Experimental studies have provided macroscopic insights into the behaviour of gapgraded soils subject to shear deformation (e.g. Salgado et al. (2000); Vallejo (2001); Carraro et al. (2009); Xiao et al. (2017)) and seepage (e.g. Skempton and Brogan (1994); Moffat et al. (2011); Ke and Takahashi (2012); Marot et al. (2016)), as well as the small strain stiffness (Wichtmann et al., 2015).

Some researchers have used three-dimensional Discrete Element Method (DEM) simulations to investigate the particle-scale mechanisms that determine the behaviour of gap-graded soils. Minh et al. (2014) simulated one-dimensional compression tests and varied the fines content in their samples to investigate the strong force chains in binary mixtures. They identified how each type of contact (i.e. contacts between fine-fine, fine-coarse and coarse-coarse particles) contributed to the overall sample stress. These observations were extended to drained triaxial compression by de Frias Lopez et al. (2016) and undrained triaxial compression by Zhou et al. (2018). Zhou et al. (2018) also investigated the induced anisotropy of the contact network using a partitioned stress-force-fabric framework (Sufian et al., 2017). Each of these DEM studies of gapgraded soils considered a single particle size ratio. Shire et al. (2014) and Shire et al. (2016) varied both the size ratio and fines content for isotropically compressed samples to investigate the fraction of stress carried by the fines.

This study uses DEM simulation data to consider the combined influence of fines content and particle size ratio on the response of gap-graded soils subject to constant mean stress $\left(p^{\prime}\right)$ triaxial compression. The contribution of the stress transmitted by different particle types (fine and coarse) and different contact types (fine-fine, fine-coarse 
and coarse-coarse) to the overall stress are considered. The anisotropy of the stress transmitted by each of these contact types is also explored. These analyses have enabled assessment of the following three key features of gap-graded soils: (i) the concept of a unique threshold fines content separating fines- and coarse-dominated behaviour; (ii) the definition of void ratio for gap-graded soils which may have non-active fine and coarse particles; and (iii) the influence of shear deformation on the proportion of fines that are unstressed and thus susceptible to seepage-induced migration (internal erosion).

As outlined in Zuo and Baudet (2015), amongst others, the threshold fines content is the fines content that differentiates gap graded materials whose behaviour is determined by the coarser grains, from materials whose behaviour is determined by the finer grains. Several methods have been suggested to identify the threshold fines content, including identifying the local minima when the experimentally determined minimum and maximum void ratios are plotted against fines content (Lade et al., 1998) or analytically using alternate void ratio definitions (Thevanayagam and Mohan, 2000; Salgado et al., 2000). Other approaches consider the intercept of the normal compression line (Carrera et al., 2011) or the critical state line (Klotz and Coop, 2002), which, in principle, provide a mechanical basis for identifying the threshold fines content. Zuo and Baudet (2015) found a large variation between the different methods, with the analytical approaches unable to predict experimental values. This extent of the discrepancy was found to depend on the size ratio of the coarser and finer grains, where it was suggested that soil fabric plays a key roles. This raises doubts on the existence of a unique threshold fines content.

Alternate definitions of void ratio have been proposed to account for the role of nonactive particles in gap-graded soils, including inter-fine, inter-granular and equivalent granular void ratio (Vaid, 1994; Thevanayagam et al., 2002). These definitions of void ratio can be used as state variables to obtain a single critical state line (Kuerbis et al., 1988; Thevanayagam and Mohan, 2000; Ni et al., 2004). However, the non-active 
particles are not readily measurable in most physical experiments. The particle-scale data from DEM simulations provides a direct route to investigate non-active particles, which will enable the definition of a granular void ratio in a consistent manner to account for both non-active fine and coarse particles.

While void ratio is a macro-scale scalar measure, it is equally important to understand the fabric arising from the application of mechanical stresses, particularly anisotropic stress conditions. Skempton and Brogan (1994) postulated that the finer fraction is more susceptible to particle transport and suffusion when transmitting a lower proportion of the applied stress, which was confirmed by Shire et al. (2014) with DEM simulations. In this study, the stress constraint on particles during anisotropic loading is investigated to show how shearing can result in complex mechanical fabrics with samples transitioning from fines- to coarse-dominated.

\section{Numerical Simulations}

The DEM simulations were performed using the open-source software LAMMPS (Plimpton, 1995). The particle size distributions considered in this study (Fig. 1) were selected to explore the influence of particle size ratio and fines content on the behaviour of gapgraded soils. The particle size ratio, $\chi=\frac{d_{p, \max }}{d_{p, \min }}$, is defined as the ratio of maximum to minimum particle diameter, with $d_{p, \max }=1 \mathrm{~mm}$ in all simulations. The fines content, $f_{c}$, is the volume fraction of finer particles with diameter $d_{p} \leq 0.5 \mathrm{~mm}$. Table 1 presents $\chi, f_{c}$, number of coarse particles $\left(N_{p}^{\mathrm{c}}\right)$, number of fine particles $\left(N_{p}^{\mathrm{f}}\right)$ and total number of particles $\left(N_{p}\right)$ for the samples considered in this study. For all samples $N_{p}^{\mathrm{c}} \approx 4000$; this value was selected following a parametric study with $N_{p}^{\mathrm{c}} \approx 500$ and $N_{p}^{\mathrm{c}} \approx 13500$ to ensure a balance between computational efficiency and achieving a representative element volume. Keeping a constant number of coarse particles is essential to ensure that subsequent analysis are not affected by differing sample sizes. Prior studies typically used $N_{p}^{\mathrm{c}}<1000$ (Minh et al., 2014; Shire et al., 2014; de Frias Lopez et al., 2016; 
Zhou et al., 2018). The larger sample sizes in this study provides greater confidence that the subsequent analysis involving subset of particles and contacts are statistically significant. With $N_{p}$ exceeding 3.2 million for the highest $\chi$ value, these simulations are amongst the largest system sizes that have been considered in published geomechanics studies (O'Sullivan, 2014) and were achieved by using distributed memory high performance computers. Moreover, on the basis of a second parametric study, the rate of shearing was selected to maintain an inertia number of $I \approx 10^{-6}$ (Da Cruz et al., 2005). The suitability of this strain rate was confirmed using simulations with $I \approx 10^{-5}$ and $I \approx 10^{-7}$. This is several orders of magnitude lower than the quasi-static limit of $I=10^{-3}$ typically mentioned in the literature, which clearly was not appropriate for the gap-graded samples considered in this study.

Three $\chi$ values were considered, 5.7, 8.0, and 14.3, having different degrees of internal instability, as quantified using the Kézdi criterion (Kézdi, 1969). The Kézdi criterion considers $\chi_{k}=\frac{d_{p, 15}^{\mathrm{c}}}{d_{p, 85}^{\mathrm{f}}}$, where $15 \%$ (by volume) of the coarse particles are smaller than $d_{p, 15}^{\mathrm{c}}$ and $85 \%$ (by volume) of fine particles are smaller than $d_{p, 85}^{\mathrm{f}}$. Following Kézdi (1969) and Sherard (1979), samples with $\chi=5.7\left(\chi_{k} \approx 3.3\right)$ are internally stable (as $\left.\chi_{k}<4.0\right)$, while samples with $\chi=14.3\left(\chi_{k} \approx 8.3\right)$ are internally unstable (as $\left.\chi>5.0\right)$. Samples with $\chi=8.0\left(\chi_{k}=4.4\right)$ are borderline unstable as $4.0 \leq \chi_{k} \leq 5.0$. The selection of size ratios also considered the limiting condition of $\chi=6.5$, which is the size ratio where a fine particle can fit within the constriction formed by the coarse particles (Lade and Yamamuro, 1997).

For each $\chi$ value, $f_{c}$ was varied between 0.18 and 0.45 to encompass the under-filled $\left(f_{c} \leq 0.24\right)$, transitional $\left(0.24<f_{c}<0.35\right)$ and over-filled $\left(f_{c} \geq 0.35\right)$ regions detailed in Shire et al. (2014), as well as the mixture classifications in Thevanayagam et al. (2002) (except the case of coarse particles fully dispersed in a fine-grained matrix, which occurs at very high fines content). In an under-filled soil, stress is primarily transmitted through the coarse particles, while stress transmission is shared between fine and coarse particles in an over-filled fabric. In the transitional region, Shire et al. 
(2014) showed a density dependence in isotropically compressed samples, where looser samples exhibited similar characteristics to an under-filled fabric, while denser samples resembled an over-filled fabric. Here, dense and loose samples were considered for each combination of $\chi$ and $f_{c}$.

The samples were prepared by isotropically compressing a non-interacting cloud of particles to a target confining pressure of $p^{\prime}=100 \mathrm{kPa}$. Periodic boundary conditions were used and gravity was neglected. The particle generation algorithm allows particles to cross the periodic boundaries so that a uniform, random distribution of particles of all sizes was attained across the periodic cell. A simplified Hertz-Mindlin contact model was used with a shear modulus of $G=29 \mathrm{GPa}$ and a Poisson ratio of $\nu=0.2$. The inter-particle friction coefficient during isotropic compression was set to $\mu=0.0$ for the dense samples and $\mu=0.3$ for the loose samples. Note that the qualitative classifications of "loose" and "dense" cannot be mapped to specific relative density values that might be considered in experimental studies. Once the target confining pressure was attained, $\mu$ was set to 0.3 and additional equilibration cycles were run at $p^{\prime}=100 \mathrm{kPa}$. No rotational resistance model was considered in this study. Constant $p^{\prime}$ triaxial compression simulations were carried out to explore the influence of the stress-induced anisotropy without considering the effects of variation in $p^{\prime}$. Shearing was terminated at an axial strain of approximately $\varepsilon_{a}=5.0 \%$ due to computational limitations associated with the large system sizes and low strain rates.

The initial void ratios, $e_{0}$, for all samples presented in Fig. 2 a show that $e_{0}$ is a function of $f_{c}, \chi$ and the density of the sample. A reduction in $e_{0}$ with increasing $f_{c}$ is observed until a local minima is reached, after which $e_{0}$ increases with increasing $f_{c}$. This reflects the fact that fines are filling the void space between the coarser particles until the minima is reached (i.e. an underfilled fabric), after which the fines start to separate the coarser particles such that the packing structure gradually transitions to coarser particles floating in a matrix of fines (i.e. an overfilled fabric). This is a wellknown observation that is in agreement with prior experimental (Lade and Yamamuro, 
1997; Thevanayagam et al., 2002) and numerical (Zhou et al., 2018; de Frias Lopez et al., 2016; Shire et al., 2016) studies. In addition, $e_{0}$ decreases with increasing $\chi$ reflecting the fact that smaller fines are better able to fill the pore space between coarser particles. The value of $f_{c}$ at which the minima in $e_{0}$ occurs shows a slight dependence on $\chi$ and a substantial dependence on the density, with the loose cases attaining the minimum $e_{0}$ value at higher $f_{c}$ values.

The macroscopic stress-strain and volumetric response (Figs. 3-4) is also affected by $f_{c}, \chi$ and density. For $\chi=5.7$ and 8.0, the response observed is typical of sand, where the dense samples exhibit post-peak strain softening and volumetric dilation, while the loose samples shows a strain hardening response with volumetric contraction. In the dense cases a clear peak deviatoric stress, $q_{\text {peak }}$, was obtained which was observed to be a function of $f_{c}$. $q_{\text {peak }}$ increased with increasing $f_{c}$ until a maximum value was reached, after which $q_{\text {peak }}$ decreased (Fig. 2b). This is reflective of the initial void ratio relationship with $f_{c}$, that is, samples with higher initial densities exhibited a higher $q_{\text {peak }}$. The maximum in $q_{\text {peak }}$ occurred at $f_{c} \approx 0.25-0.30$, which is in close agreement with the experimental observations of Vallejo (2001), where direct shear tests were conducted on binary mixtures of spherical glass beads and peak shear strength was observed for fines content of $f_{c} \approx 0.20-0.25$ at various normal pressures. The postpeak strain softening response is also influenced by $f_{c}$, with samples with higher $e_{0}$ exhibiting a higher degree of softening.

For $\chi=14.3$, the volumetric response is similar to that observed for other particle size ratios, i.e. dense samples dilated while loose samples contracted. However, the stress-strain response differed substantially. For the dense cases, only $f_{c}=0.18,0.25$ showed a stiff initial increase in $q$ that was noted for all dense cases in $\chi=5.7,8.0$, while for $f_{c}=0.30,0.35,0.45$ a more gradual increase in $q$ is noted. For the dense cases at $\chi=14.3$ for $f_{c}=0.18,0.25,0.30, q_{\text {peak }}$ is obtained at larger axial strains with minimal post-peak strain softening. For $f_{c}=0.35,0.45$, peak deviatoric stress was not attained at the end of simulation, and hence, is not plotted in Fig. 2b. When 
considering $q_{\text {peak }}$ for $\chi=14.3$, a dependency on $\chi$ is noted; $q_{\text {peak }}$ increases from $\chi=5.7$ to $\chi=8.0$, and then reduces when $\chi=14.3$, while $f_{c}$ at which the maximum occurs reduces with increasing $\chi$.

In contrast to the observations for $\chi=5.7$ and 8.0, the magnitude of $q$ shows a significant reduction at higher fines content $\left(f_{c}=0.35\right.$ and 0.45$)$ for $\chi=14.3$. The over-filled fabric at higher $f_{c}$ implies that the stress is shared between the finer and coarser fraction, while the higher $\chi$ indicates a larger number of fine particles separating the coarser fraction. For these conditions, it is hypothesised that the change in the topology of the force chain network results in force chains more readily changing during shearing leading to the significant reduction in $q$. These data suggest a need for further DEM and experimental exploration of the response of gap-graded soils at similar $f_{c}$ and $\chi$ under drained conditions.

A strain hardening response is noted in all loose cases with the exception of the $\chi=14.3, f_{c}=0.30$ case in Fig. $3(\mathrm{f})$, which exhibited an initial metastable response. This metastable response was confirmed to be repeatable in three different simulations for this case using different initial particle positions. Consequently, the stress-strain response in Fig. 3(f) (and subsequent figures) for this case is shown as a dotted line and results should be interpreted with caution.

The data presented in Fig. 2 indicate a change in the material structure at particular $f_{c}$ values. However, both $q_{\text {peak }}$ and $e_{0}$ exhibit local maximum/minimum at a different $f_{c}$ for a given $\chi$. This confirms the comments of Zuo and Baudet (2015) and raises questions as to the validity of a unique threshold fines content $\left(f_{c}^{\text {thres }}\right)$ delineating fineand coarse-dominated behaviour. These observations motivate a more detailed analysis of fabric that follows. 


\section{Distribution of Stress in Fine and Coarse Par-} ticles

\subsection{Particle-based Stress Partition}

The relative influence of the fine and coarse particles on the mechanical response can be attained by looking at the contribution of each class of particle to the overall stress transmitted. The stress tensor for an individual particle, $\sigma_{i j}^{\mathrm{m}}$, is defined as (Potyondy and Cundall, 2004):

$$
\sigma_{i j}^{\mathrm{m}}=\frac{1}{V^{\mathrm{m}}} \sum_{\mathrm{c}=1}^{N_{\mathrm{c}}^{\mathrm{m}}} f_{j}^{\mathrm{c}}\left(x_{i}^{\mathrm{c}}-x_{i}^{\mathrm{m}}\right)
$$

where $V^{\mathrm{m}}$ is the particle volume, $N_{\mathrm{c}}^{\mathrm{m}}$ is the number of contacts involving the particle, $\mathbf{f}^{\mathrm{c}}$ is the contact force vector, $\mathbf{x}^{\mathrm{c}}$ is the position of the contact point and $\mathbf{x}^{\mathrm{m}}$ is the position of the particle centroid. The stress tensor for the entire assembly of particles, $\sigma_{i j}$, can be calculated as:

$$
\sigma_{i j}=\frac{1}{V} \sum_{\mathrm{m}=1}^{N_{p}} V^{\mathrm{m}} \sigma_{i j}^{k}
$$

where $V$ is the assembly volume. $\sigma_{i j}$ can be decomposed into contribution of the finer $\left(\sigma_{i j}^{\mathrm{f}}\right)$ and coarser $\left(\sigma_{i j}^{\mathrm{c}}\right)$ fractions:

$$
\sigma_{i j}=\underbrace{\frac{1}{V} \sum_{\mathrm{m}=1}^{N_{p}^{\mathrm{f}}} V^{\mathrm{m}} \sigma_{i j}^{k}}_{\sigma_{i j}^{\mathrm{f}}}+\underbrace{\frac{1}{V} \sum_{\mathrm{m}=1}^{N_{p}^{\mathrm{c}}} V^{\mathrm{m}} \sigma_{i j}^{k}}_{\sigma_{i j}^{\mathrm{c}}}
$$

The proportion of stress carried by the finer fraction is given by:

$$
\alpha_{\sigma}=\frac{\sigma^{\mathrm{f}}}{\sigma}
$$

where $\sigma^{\mathrm{f}}$ denotes a stress quantity for the finer fraction and $\sigma$ denotes a stress quantity for the entire assembly. For example, if the stress quantity of interest is the mean stress 
$(p)$, then $\alpha_{p}=\frac{p^{\mathrm{f}}}{p}$, where $p^{\mathrm{f}}=\frac{\sigma_{i i}^{\mathrm{f}}}{3}$ from Eq. 3 and $p=\frac{\sigma_{i i}}{3}$ in Eq. 2. The proportion of deviatoric stress $(q)$ carried by the finer fraction, $\alpha_{q}$, can be calculated in a similar manner. Eq. 4 is conceptually similar to the stress reduction factor in Shire et al. (2014), but $0 \leq \alpha_{\sigma} \leq 1$, which allows for a more intuitive understanding of how stress is partitioned between the finer and coarser fractions.

Fig. 5 shows the variation in $\alpha_{p}$ with $f_{c}$ at the start and end of shearing, while Fig. 6 plots the evolution of $\alpha_{p}$ during triaxial shearing. Similar trends were observed for $\alpha_{q}$ (not shown here). For a given $\chi, \alpha_{p}$ increases with increasing $f_{c}$ at all strain levels considered. For all $\chi$ values considered, $\alpha_{p}<0.10$ for $f_{c}=0.18$, indicating that less than $10 \%$ of the mean stress is carried by the finer fraction, while $\alpha_{p}>0.35$ for $f_{c}=0.45$. Intuitively, the finer particles play a more active stress-carrying role as the proportion of fines increases to fill the void spaces, confirming the delineation between under-filled and over-filled gap-graded soils. Unlike $q_{\text {peak }}$ and $e_{0}$ in Fig. 2, where a local maxima/minima was observed, Fig. 5 shows that $\alpha_{p}$ increases monotonically with $f_{c}$ and this gradual transition from under-filled to over-filled fabric does not support the concept of a unique $f_{c}^{\text {thres }}$.

The relationship between $\alpha_{p}$ and $\chi$ can most easily be seen by reference to Fig. 5 . For low $f_{c}$ values (i.e. $\left.f_{c}=0.18,0.25\right), \alpha_{p}$ decreases with increasing $\chi$. This reflects the fact that the lowest $\chi$ value considered is less than the limit of $\chi=6.5$ proposed by Lade and Yamamuro (1997), which is the size ratio where a fine particle can fit within the constriction formed by three coarse particles. As $\chi$ increases the finer particles are better able to fit within the pore space of the coarse matrix without transmitting stress. For $f_{c}=0.30,0.35$, more complex trends emerge; $\alpha_{p}$ initially decreases as $\chi$ increases from 5.7 to 8.0 and subsequently increases as $\chi$ increases from 8.0 to 14.3. For high fines content (e.g. $\left.f_{c}=0.45\right), \alpha_{p}$ increases with increasing $\chi$, which reflects the fact that at a given $f_{c}$ value, a greater number of fine grains separate the the coarse fractions when $\chi$ is larger, so that the relatively smaller fine particles play a 
more prominent role in carrying the applied stress.

Density has a significant influence on the redistribution of stress during shearing (Fig. 6). The loose cases show minimal changes in $\alpha_{p}$, with only a few cases exhibiting a slight increase. In contrast, the dense cases show significant reduction in $\alpha_{p}$ during shearing when $f_{c}>0.18$. The reduction in $\alpha_{p}$ is most prominent in the dense transitional cases (e.g. $f_{c}=0.25,0.30,0.35$ in Figs. 6(a)-(c)). In the clearly under-filled fabrics, i.e. $f_{c}=0.18$, the variation in $\alpha_{p}$ with $\varepsilon_{a}$ is negligible, while in clearly overfilled fabrics, i.e. $f_{c}=0.45, \alpha_{p}$ initially exhibits a slight reduction and then appears to attain a steady state value at $\varepsilon_{a}<0.5 \%$

These observations demonstrate that for dense assemblies with transitional $f_{c}$ values, the shearing process induces a shift in the proportion of stress transmitted by each size fraction, such that stress transmission by the coarser particles becomes more dominant. The data further support the argument that it is misleading to consider that a single $f_{c}^{\text {thres }}$ value suffices to judge whether the coarser or finer fraction dominates the mechanical response of a gap-graded material, with both density and stress anisotropy playing a key role.

\subsection{Stresses on Individual Particles}

$\alpha_{p}$ is an averaged quantity describing the contribution of the finer fraction to the mean stress of the assembly; it does not provide insight into the range of stresses transmitted by each size fraction. Fig. 7 shows the cumulative distribution (by volume) of the normalised mean stress on individual particles, $\frac{p^{\mathrm{m}}}{p}$, at the start and end of shearing. The distribution for the finer and coarser fractions are shown separately. Only the dense cases are shown in Fig. 7, as these cases exhibited the most pronounced changes in $\alpha_{p}$.

A broad range of particle mean stress is observed in the finer fraction, which is masked when considering the averaged quantity $\alpha_{p}$. For example, in the dense $f_{c}=$ 
$0.25, \chi=8.0$ case, $\alpha_{p} \approx 0.18$ (Fig. 5a) at the start of shearing, but approximately $30 \%$ of fines have a mean stress greater than the assembly mean stress (Fig. 7 a(ii)). Conceptual models of gap-graded soils often assume that the fines do not carry any stress for $f_{c}<f_{c}^{\text {thres }}$, but the distributions shown in Fig. 7a indicates that this is only the case at large particle size ratios.

The proportion of non-active fines with $\frac{p^{\mathrm{m}}}{p} \approx 0$ (illustrated by the vertical drop in the distribution at $\frac{p^{\mathrm{m}}}{p} \approx 0$ ) increased during shearing for all $f_{c}$ and $\chi$. A substantial increase in non-active fines was noted for $f_{c}=0.25$, with the proportion of nonactive fines increasing from $20 \%$ at the start of shearing to exceeding $80 \%$ at the end of shearing (Fig. 7a). For $f_{c}=0.18,0.25,0.30$, the proportion of non-active fines increased with increasing $\chi$, while for $f_{c}=0.35,0.45$ the proportion of non-active fines reduced with increasing $\chi$. The proportion of the finer fraction carrying a mean stress above the assembly mean stress (i.e. $\frac{p^{\mathrm{m}}}{p}>1$ ) increased with increasing fines content from approximately $5 \%$ for $f_{c}=0.18$ to $70 \%$ for $f_{c}=0.45$. This proportion reduced during shearing and by the end of shearing less than $5 \%$ of fines have above the assembly mean stress for $f_{c}=0.18,0.25$, while for $f_{c}=0.45$ approximately $45 \%$ of fines carry above-average mean stress (Fig. 7a).

For the coarser fraction, the breadth of the distribution reduced with increasing $f_{c}$. For $f_{c}=0.45$, coarse particles predominantly exhibit a mean stress between $0.9 p \leq$ $p^{\mathrm{m}} \leq 1.2 p$ (for all $\chi$ ), while for $f_{c}=0.18$ the range of individual forces on the coarse particles ranges from $p^{\mathrm{m}}=0$ to $p^{\mathrm{m}} \geq 3.0 p$ (Fig. $7 \mathrm{~b}$ ). The breadth of the distribution for the coarse particles broadens and shifts to the right with shearing, reflecting the greater proportion of the overall stress that is being transmitted by the coarser fraction. This reflects the increase in the non-active fines noted in Fig. 7a and the reduction in $\alpha_{p}$ noted in Fig. 6. In agreement with the observations for $\alpha_{p}$, it is not possible to identify a unique $f_{c}^{\text {thres }}$ value that is associated with a distinct change in the shape of the distribution. 


\subsection{Alternative Void Ratio Definitions}

In granular materials with non-active fine particles, a number of authors have suggested that these particles should be considered as part of the void space, to enable application of correlations including void ratio to gap-graded soils. Referring to earlier contributions including Vaid (1994) and Thevanayagam et al. (2002), various alternative definitions of void ratio were considered to account for non-active particles. The inter-granular void ratio, $e_{c}$, which considers all fine particles to be non-active in the stress transmission process and part of the pore space is given by:

$$
e_{c}=\frac{e+f_{c}}{1-f_{c}}
$$

This is applicable to gap-graded soils with low fines content, such that the under-filled structure results in fines located within the pores of a coarse matrix.

The inter-fine void ratio, $e_{f}$, which considers all coarse particles to be non-active, is given by:

$$
e_{f}=\frac{e}{f_{c}}
$$

Note that $e_{f}$ does not consider the non-active coarse particles to be part of the void space, but rather they have have zero contribution to the solid volume. This definition is applicable for gap-graded soils at high fines content, such that the over-filled structure results in coarse particles floating in a dispersed manner within a matrix of fine particles.

To account for the combined contribution of the finer and coarser fractions, the equivalent granular void ratio $\left(e_{e q}\right)$ is defined as:

$$
e_{e q}=\frac{e+(1-b) f_{c}}{1-(1-b) f_{c}}
$$

where $b$ is the proportion of active stress transmitting fines. When $b=1$, all fines are contributing to stress transmission and $e_{e q}=e ; b=0$ implies none of the fines are active 
and $e_{e q}=e_{c}$. If $0 \leq b \leq 1$, the equivalent granular void ratio, $e_{e q}$, cannot describe a fines-dominated matrix, and hence, the inter-fine void ratio cannot be recovered. Obtaining $b$ from experimental studies is challenging and is typically done by backcalculation using correlations between $e_{e q}$ and the measured steady-state shear strength (Rahman et al., 2008). A semi-empirical relationship for $b$ using experimental results on binary packings was proposed in Rahman et al. (2008), where $b$ is expressed as a function of the particle size ratio and fines content.

Discrete element simulations provide a direct route to calculate $b$ using the measured particle stresses in Sec. 3.2. The proportion of non-active fine particles is given by:

$$
\eta_{\mathrm{f}}=\frac{V_{s}^{\mathrm{f}}\left(p \leq p^{*}\right)}{V_{s}}
$$

where $V_{s}^{\mathrm{f}}\left(p \leq p^{*}\right)$ is the volume of fine particles with mean stress below the threshold $p^{*}$ and $V_{s}$ is the volume of all particles. This is directly related to the $b$ parameter in Eq. 7:

$$
b=1-\frac{\eta_{\mathrm{f}}}{f_{c}}
$$

The proportion of non-active coarse particles is given by:

$$
\eta_{\mathrm{c}}=\frac{V_{s}^{\mathrm{c}}\left(p \leq p^{*}\right)}{V_{s}}
$$

where $V_{s}^{\mathrm{c}}\left(p \leq p^{*}\right)$ is the volume of coarse particles with mean stress below the threshold. Assuming that non-active fines are part of the void space, while non-active coarse particles have zero solid volume contribution (as per the assumptions in Thevanayagam et al. (2002)), a granular void ratio $\left(e_{g}\right)$ is defined:

$$
e_{g}=\frac{e+\eta_{\mathrm{f}}}{1-\eta_{\mathrm{f}}-\eta_{\mathrm{c}}}
$$

If all fines are non-active (i.e. for low $f_{c}$ ), then $\eta_{\mathrm{f}}=f_{c}$ and $\eta_{\mathrm{c}}=0$, and Eq. 11 collapses 
to the limiting condition of the inter-granular void ratio in Eq. 5. If all the coarse particles are non-active (i.e. at high $f_{c}$ ), then $\eta_{\mathrm{f}}=0$ and $\eta_{\mathrm{c}}=1-f_{c}$, and Eq. 11 recovers the inter-fine void ratio in Eq. 6. Hence, $e_{g}$ in Eq. 11 provides a consistent way to consider the contribution of active fine and coarse particles for all fines content. The choice of the threshold stress can be adjusted and Fig. 8 shows the influence of various threshold parameters ranging from $p^{*}=1 \mathrm{~Pa}$ to $p^{*}=1000 \mathrm{~Pa}$. As expected, $e_{g}$ increases as the threshold mean stress increases. However, the difference is minimal for most cases with the exception of $f_{c}=0.30$.

Fig. 9 presents the ratio of granular void ratio to macroscopic void ratio $\left(e_{g} / e\right)$ at the start and end of shearing. $1.0 \leq e_{g} / e \leq 3.0$ at the start of shearing, with the loose cases exhibiting the most substantial increase in $e_{g}$, indicating a significant portion of non-active fine and coarse particles. An approximately concave relationship is noted for the loose cases in Fig. 9a, with the maximum increase observed for $f_{c}=0.25,0.30$. The dense assemblies only exhibited a small increase with $e_{g} \approx 1.1 e$, except for the $f_{c}=0.18$ case where $e_{g} \approx 1.7 e$ at the start of shearing. At the end of shearing, Fig. $9 \mathrm{~b}$ shows a notable increase in $e_{g}$ for the dense cases, which is supported by increasing value of $\eta_{\mathrm{f}}$ and $\eta_{\mathrm{c}}$ observed in the distributions in Fig. 7 . The loose cases show minimal change in $e_{g}$ with shearing.

Fig. 10 shows the evolution of $e_{g}$ with shearing for the dense cases only using a threshold mean stress of $p^{*}=1 \mathrm{~Pa} . e_{g}>e$ throughout shearing, as non-active fine particles are being added to the void space, while non-active fine and coarse particle volumes are removed from the total particle volume with strain. Nevertheless, $e_{g}$ broadly captures the expected macroscopic volumetric response with dilation in the dense cases and slight contraction in the loose cases. The rate of increase in $e_{g}$ differs from the macroscopic dilation rate. In particular, the $f_{c}=0.25$ cases exhibit a very large increase in $e_{g}$, which is in line with the observations in Fig. 7, where a clear increase in non-active fines is noted. This is also captured in the $b$ parameter, which is calculated using Eq. 9 and plotted in Fig. 11 for the dense cases only. $b$ decreases with 
strain, as stress is redistributed from the finer to coarser fraction during shearing.

As evident in the $\alpha_{p}$ parameter, this reduction in $b$ was most prominent in the dense transitional cases. This suggests that there is a strong density dependence on the redistribution of stress and to explore this, Fig. 12 plots the correlation between $\alpha_{p}$ and both the traditional definition of $e$ and the granular void ratio $e_{g}$ introduced above. While limited correlation can be observed when considering $e$, there is a much better correlation between $\alpha_{p}$ and $e_{g}$. For each combination of $\chi$ and $f_{c}$, Fig. 12b shows that the $\alpha_{p}$ vs. $e_{g}$ relationship for the loose and dense samples can be joined together by a single expression (with the exception of $\chi=14.3, f_{c}=0.30$ which was metastable). This indicates that $e_{g}$ is a useful parameter to investigate the contribution of two size fractions to the stress transmitted through a gap-graded. However, it is clear that the relationship is not unique when considering all fines content and particle size ratios. This suggest that a more detailed investigation is required to understand how the two size fractions interact in transmitting stress in gap-graded soils and the associated fabric anisotropy.

\section{Distribution of Stress in Inter-particle Con-}

\section{tacts}

\subsection{Contact-based Stress Partition}

The $\alpha$ parameter provides little insight into the interaction of the two size fractions, however this can be investigated by analysing the inter-particle contact network. A contact-based stress partition can be obtained if we consider that the assembly stress tensor can be obtained from the inter-particle contacts:

$$
\sigma_{i j}=\frac{1}{V} \sum_{n=1}^{N_{c}} f_{i}^{c} l_{j}^{c}
$$


where $\mathbf{f}^{c}$ is the contact force vector, $\mathbf{l}^{c}$ is the branch length vector, and the summation is over all $N_{c}$ contacts in the domain. $\sigma_{i j}$ can be decomposed to identify the contributions of each contact class, that is, contact between (i) fine-fine; (ii) fine-coarse; and (iii) coarse-coarse particles, denoted $f f, f c, c c$, respectively. The stress tensor for each contact partition, $\sigma_{i j}^{\mathrm{k}}$, is determined by:

$$
\sigma_{i j}^{\mathrm{k}}=\frac{1}{V} \sum_{n=1}^{N_{c}^{\mathrm{k}}} f_{i}^{c} l_{j}^{c}
$$

where $N_{c}^{\mathrm{k}}$ is the number of contacts within the specified partition $\mathrm{k} \in\{\mathrm{ff}, \mathrm{fc}, \mathrm{cc}\}$ and $\sigma_{i j}=\sum_{\mathrm{k}} \sigma_{i j}^{\mathrm{k}}$. In a similar manner to Eq. 4, the proportion of a stress quantity carried by contact partition $\mathrm{k}$ is given by:

$$
\beta_{\sigma}^{\mathrm{k}}=\frac{\sigma^{\mathrm{k}}}{\sigma}
$$

where $\sigma^{\mathrm{k}}$ denotes a stress quantity for a partition and $\sigma$ denotes the same stress quantity for the entire assembly. $\beta_{\sigma}^{\mathrm{ff}}+\beta_{\sigma}^{\mathrm{fc}}+\beta_{\sigma}^{\mathrm{cc}}=1.0$, and hence there are only two independent terms when considering the $\mathrm{k} \in\{\mathrm{ff}$, fc, cc $\}$ partition. Fig. 13 shows the evolution of $\beta_{p}^{\mathrm{k}}$ for the dense cases only (as the loose cases exhibited minimal changes in $\beta_{p}^{\mathrm{k}}$ during shearing).

At all strain levels the proportion of mean stress transmitted through the fine-fine $\left(\beta_{p}^{\mathrm{ff}}\right)$ and fine-coarse $\left(\beta_{p}^{\mathrm{fc}}\right)$ contacts increases with increasing $f_{c}$, and consequently the stress transmitted through the coarse-coarse fraction decreases with increasing $f_{c}$. At higher fines content $\left(f_{c}=0.35,0.45\right)$, a maximum value in $\beta_{p}^{\mathrm{fc}}$ is attained, indicating an upper limit to the amount of stress transmitted through the contacts formed by the interaction of the fine and coarse particles.

The distribution of stress is clearly sensitive to $\chi$. At low fines contents (e.g. $\left.f_{c}=0.18,0.25\right)$, increasing $\chi$ generally results in a reduction in $\beta_{p}^{\mathrm{ff}}$ and $\beta_{p}^{\mathrm{fc}}$, while $\beta_{p}^{\mathrm{cc}}$ increases. At high fines content (e.g. $\left.f_{c}=0.30,0.35,45\right), \beta_{p}^{\mathrm{ff}}$ and $\beta_{p}^{\mathrm{fc}}$ both increase 
with increasing $\chi$, while $\beta_{p}^{\text {cc }}$ decreases with increasing $\chi$.

Shearing results in a redistribution of stress from the fine-fine and fine-coarse contacts to the coarse-coarse contacts. This is most apparent in the transitional cases (i.e. $\left.f_{c}=0.25,0.30,0.35\right)$, where there is a substantial change in $\beta_{p}^{\mathrm{k}}$ with strain. This is in agreement with the observations in $\alpha_{p}$ and confirms that dense transitional cases are most susceptible to redistribution in stress during shearing. $\beta_{p}^{\mathrm{cc}}$ is the most suitable parameter to investigate the redistribution of stress as is covers the complete range $0.0 \leq \beta_{p} \leq 1.0$ and so Figure 14 considers $\beta_{p}^{\text {cc }}$ at the start and end of shearing. A minimal degree of stress redistribution is noted in the loose cases, while in the dense transitional cases, there is a clear upward shift in $\beta_{p}^{\mathrm{cc}}$. Moreover, a gradual transition in $\beta_{p}^{c c}$ is noted with fines content, and hence, a unique $f_{c}^{\text {thres }}$ cannot be identified.

$\alpha_{p}$ only allows for the distinction between an assembly which in dominated by fines or coarse. In contrast, $\beta_{p}$ allows for four categories of material to be distinguished:

1. $\beta_{p}^{\mathrm{cc}} \geq \beta_{p}^{\mathrm{fc}} \geq \beta_{p}^{\mathrm{ff}}:$ coarse-dominated $(\mathrm{C})$

2. $\beta_{p}^{\mathrm{fc}} \geq \beta_{p}^{\mathrm{cc}} \geq \beta_{p}^{\mathrm{ff}}:$ transitional coarse-dominated (TC)

3. $\beta_{p}^{\mathrm{fc}} \geq \beta_{p}^{\mathrm{ff}} \geq \beta_{p}^{\mathrm{cc}}:$ transitional fines-dominated $(\mathrm{TF})$

4. $\beta_{p}^{\mathrm{ff}} \geq \beta_{p}^{\mathrm{fc}} \geq \beta_{p}^{\mathrm{cc}}$ : fines-dominated $(\mathrm{F})$

A further two categories are possible (i.e. $\beta_{p}^{\mathrm{cc}} \geq \beta_{p}^{\mathrm{ff}} \geq \beta_{p}^{\mathrm{fc}}$ and $\beta_{p}^{\mathrm{ff}} \geq \beta_{p}^{\mathrm{cc}} \geq \beta_{p}^{\mathrm{fc}}$ ), but these rarely physically attainable, and hence, are not included above. Fig. 15 illustrates how both $f_{c}$ and $\chi$ determine where dense dilating assemblies transition to becoming dominated by the coarse fraction.

\subsection{Anisotropy of the Inter-Particle Contact Network}

In addition to the stress transmitted through the contact network, the evolution of the material directional fabric anisotropy during shearing is required to develop a full understanding of how the two size fractions interact. The anisotropy was investigated 
using the partitioned stress-force-fabric (SFF) framework (Sufian et al., 2017), which relates the macroscopic stress to micro-scale anisotropy parameters. This framework is applied to the three sets of contacts (fine-fine, fine-coarse and coarse-coarse). The partitioned SFF relationship is given by:

$$
\frac{q}{p}=\frac{2}{5} \sum_{\mathrm{k}} \xi_{\mathrm{k}}\left(a_{c}^{\mathrm{k}}+a_{n}^{\mathrm{k}}+\frac{3}{2} a_{t}^{\mathrm{k}}+a_{b}^{\mathrm{k}}\right)
$$

where $\frac{q}{p}$ is the macroscopic stress ratio, $a_{c}^{\mathrm{k}}$ is the geometric anisotropy of the unit contact normals, $a_{n}^{\mathrm{k}}$ is the anisotropy in the normal force magnitude, $a_{t}^{\mathrm{k}}$ is the anisotropy in the tangential force magnitude, $a_{b}^{\mathrm{k}}$ is the anisotropy in the length of the branch vector, $\xi_{\mathrm{k}}$ is a weighting parameter reflecting the number of contacts, average force and average branch vector length of the $\mathrm{k}^{\text {th }}$ partition and the summation is over all $\mathrm{k}$ partitions. The anisotropy parameters in Eq. 15 can be obtained directly from discrete element simulations, as detailed in Sufian et al. (2017). As per Sec. 4.1, $\mathrm{k} \in\{\mathrm{ff}, \mathrm{fc}, \mathrm{cc}\}$, noting that an absence of any value for $\mathrm{k}$ denotes a quantity calculated for the complete contact network.

Fig. 16 shows the evolution of the various anisotropy parameters during shearing for a typical case $\left(\chi=8.0, f_{c}=0.35\right)$. Both loose and dense cases are shown. The complete contact network is considered, along with each of the $\{\mathrm{ff}, \mathrm{fc}, \mathrm{cc}\}$ subnetworks separately.

Anisotropy in the normal force magnitude $\left(a_{n}\right)$ is clearly the dominant form of anisotropy when considering the complete contact network. This is in agreement with prior observations for disks (Rothenburg and Bathurst, 1989), spherical (Sufian et al., 2017) and polyhedral (Azéma et al., 2009) particles. The strong induced anisotropy implies that the magnitude of normal force is larger in the direction of compressive loading. $a_{n}$ is also the dominant anisotropy contributor in the $\{\mathrm{ff}, \mathrm{fc}\}$ subnetworks.

The geometric anisotropy in the contact normal orientation $\left(a_{c}\right)$ is the other main contributor, but is substantially smaller than $a_{n}$ in the complete contact network. 
This implies that the contact normals show a slight preference to be orientated in the direction of compressive loading. A slight degree of anisotropy in $a_{c}$ is also noted in the $\{\mathrm{ff}, \mathrm{fc}\}$ subnetworks, but there is significant departure from this trend in the coarse-coarse network. Initially, $a_{n}>a_{c}$ in the coarse-coarse subnetwork, but with shearing $a_{c}>a_{n}$. This indicates that shear strength was predominantly provided through redistribution of force without a significant change to the contact network topology in the early stages of loading, but with shearing, sufficient re-alignment of the coarse-coarse network provided the shortest path to transmit stress.

The magnitude of anisotropy increases from the $\{\mathrm{ff}\}$ to $\{\mathrm{cc}\}$ subnetworks. For gap-graded soils, this suggests that strong force chains (Minh et al., 2014) are formed passing through the coarse-coarse network, which are braced laterally by fine-coarse contacts.

The anisotropy induced in the dense case is larger than the loose case. This is evident in the complete network and each of the subnetworks for all anisotropy parameters and is reflective of the higher shear strength attained in the dense samples. The higher degree of anisotropy in the dense samples also suggests that stress redistribution is more prevalent in highly anisotropic structures. Tangential force anisotropy $\left(a_{t}\right)$ and anisotropy in branch vector length $\left(a_{b}\right)$ have much smaller negligible contributions, with the exception of the coarse-coarse contacts.

The coarse-coarse subnetwork exhibited the most dominant characteristics in the typical case shown in Fig. 16, and consequently, the $a_{n}^{\mathrm{cc}}$ and $a_{c}^{\mathrm{cc}}$ parameters in the partitioned SFF relationship are explored in further detail in Fig. 17. $a_{n}^{\mathrm{cc}}$ and $a_{c}^{\mathrm{cc}}$ generally increase with increase $f_{c}$. This is intuitive, as there are relatively fewer coarse particles with increasing fines content, and these are required to form highly anisotropic structures to transmit stress the bulk of the shear stress. Moreover, Fig. 17 clearly demonstrates that the magnitude of anisotropy in the coarse-coarse contacts increases with $\chi$ and this is particularly prevalent for $\chi=14.3$. 


\section{Conclusions}

This study investigated the stress distribution in sheared cohesionless gap-graded soils using numerical simulations using DEM simulations. The fines content was varied to capture the under-filled, transitional and over-filled fabric states, while various particle size ratios were considered to capture different states of internal instability, including stable, borderline unstable and unstable following the Kézdi criterion. Samples were prepared by isotropically compressing a non-interacting cloud of particles to a target confining pressure, after which the samples were sheared at constant mean stress condition. Dense and loose samples were considered for each combination of fines content and particle size ratio. An emphasis was placed on simulation quality and the appropriate simulation system sizes, as established via parametric studies, were larger than in prior DEM studies of gap-graded materials. A parametric study also revealed that the strain rate (inertia number) required to remove strain rate sensitivity and achieve quasi-static simulations was significantly lower than would be predicted using values recommended in the literature.

The resulting data have enabled analyses to provide new insight into the behaviour of gap graded materials, as follows:

1. The observations in this study do not support the presence of a unique threshold fines content that can differentiate between fine and coarse dominated behaviour in gap graded soils. Consideration of particle- and contact-based partitioning of the stress revealed a gradual transition between under-filled fabrics dominated by coarse particles and over-filled fabrics where stress is shared between the finer and coarser fraction. While prior studies considered a local minima/maxima to define a threshold fines content, no defining feature could be identified when observing stress characteristics in the finer and coarser fraction. Moreover, the gradual transition in stress carried by the two fractions exhibited a density and stress anisotropy dependence that has not been considered in prior studies. Fig. 15 
provides a preliminary framework to identify transition from fines-dominated to coarse-dominated behaviour and further investigation is required to explore this framework.

2. Dense, transitional gap-graded soils exhibited a significant reduction in the stress carried by the finer fraction during shearing. Assemblies which initially shared stress between the finer and coarser fractions became dominated by the coarser fraction under anisotropic loading. The stress distribution in dense, transitional gap-graded soils was also linked to the anisotropy of the contact network both in terms of the orientation of contact normals and the normal force magnitude using the partitioned stress-force-fabric relationship.

3. Detailed insight into the stress distribution where obtained by exploring the distribution of individual particle stresses. This revealed that shearing resulted in an increase in non-active fines irrespective of the fines content, particle size ratio and density, while a broadening and shift in the distribution of stress in the coarse particles was noted. These features are masked when considering averaged stress quantities, and hence, the distribution of per-particle stress is crucial to obtain this insight. This motivated the development of an expression for the granular void ratio (Eq. 11), which incorporated the proportion of non-active fine and coarse particles. The inter-granular and inter-fine void ratio detailed in the literature are limits to the definition of granular void ratio in Eq. 11. Moreover, the $b$ parameter that is widely used in the definition of equivalent granular void ratio was not observed to be a constant and varied during shearing.

\section{Acknowledgements}

This study was funded by EPSRC grant EP/P010393/1. This work used the Imperial College London High Performance Computing facility. This work used the ARCHER 
UK National Supercomputing Service (http://www.archer.ac.uk).

\section{Data Availability Statements}

Some or all data, models, or code that support the findings of this study are available from the corresponding author upon reasonable request.

\section{References}

Azéma, E., Radjai, F., and Saussine, G. Quasistatic rheology, force transmission and fabric properties of a packing of irregular polyhedral particles. Mechanics of Materials, 41(6):729-741, 2009.

Carraro, J. A. H., Prezzi, M., and Salgado, R. Shear strength and stiffness of sands containing plastic or nonplastic fines. Journal of geotechnical and geoenvironmental engineering, 135(9):1167-1178, 2009.

Carrera, A., Coop, M., and Lancellotta, R. Influence of grading on the mechanical behaviour of stava tailings. Géotechnique, 61(11):935, 2011.

Da Cruz, F., Emam, S., Prochnow, M., Roux, J.-N., and Chevoir, F. Rheophysics of dense granular materials: Discrete simulation of plane shear flows. Physical Review E, 72(2):021309, 2005.

de Frias Lopez, R., Silfwerbrand, J., Jelagin, D., and Birgisson, B. Force transmission and soil fabric of binary granular mixtures. Géotechnique, 66(7):578-583, 2016.

Ke, L. and Takahashi, A. Strength reduction of cohesionless soil due to internal erosion induced by one-dimensional upward seepage flow. Soils and Foundations, 52(4):698$711,2012$. 
Kézdi, Á. Increase of protective capacity of flood control dikes. Technical Report 1, Department of Geotechnique. Technical University, Budapest, Hungary, 1969.

Klotz, E. and Coop, M. On the identification of critical state lines for sands. Geotechnical Testing Journal, 25(3):289-302, 2002.

Kuerbis, R., Negussey, D., and Vaid, Y. Effect of gradation and fines content on the undrained response of sand. Geotechnical special publication, (21):330-345, 1988.

Lade, P. V. and Yamamuro, J. A. Effects of nonplastic fines on static liquefaction of sands. Canadian Geotechnical Journal, 34(6):918-928, 1997.

Lade, P. V., Liggio, C., Yamamuro, J. A., et al. Effects of non-plastic fines on minimum and maximum void ratios of sand. Geotechnical testing journal, 21:336-347, 1998.

Marot, D., Rochim, A., Nguyen, H.-H., Bendahmane, F., and Sibille, L. Assessing the susceptibility of gap-graded soils to internal erosion: proposition of a new experimental methodology. Natural Hazards, 83(1):365-388, 2016.

Minh, N., Cheng, Y., and Thornton, C. Strong force networks in granular mixtures. Granular Matter, 16(1):69-78, 2014.

Moffat, R., Fannin, R. J., and Garner, S. J. Spatial and temporal progression of internal erosion in cohesionless soil. Canadian Geotechnical Journal, 48(3):399-412, 2011.

Ni, Q., Tan, T., Dasari, G., and Hight, D. Contribution of fines to the compressive strength of mixed soils. Géotechnique, 54(9):561-569, 2004.

O'Sullivan, C. Advancing geomechanics using dem. In Proc. International Symposium on Geomechanics from Micro to Macro, volume 1, pages 21-32, 2014.

Plimpton, S. Fast parallel algorithms for short-range molecular dynamics. Journal of computational physics, 117(1):1-19, 1995. 
Potyondy, D. O. and Cundall, P. A bonded-particle model for rock. International journal of rock mechanics and mining sciences, 41(8):1329-1364, 2004.

Rahman, M. M., Lo, S., and Gnanendran, C. On equivalent granular void ratio and steady state behaviour of loose sand with fines. Canadian Geotechnical Journal, 45 (10):1439-1456, 2008.

Rothenburg, L. and Bathurst, R. Analytical study of induced anisotropy in idealized granular materials. Geotechnique, 39(4):601-614, 1989.

Sail, Y., Marot, D., Sibille, L., and Alexis, A. Suffusion tests on cohesionless granular matter: Experimental study. European Journal of Environmental and Civil Engineering, 15(5):799-817, 2011.

Salgado, R., Bandini, P., and Karim, A. Shear strength and stiffness of silty sand. Journal of Geotechnical and Geoenvironmental Engineering, 126(5):451-462, 2000.

Sherard, J. Sinkholes in dams of coarse, broadly graded soils. In Proceedings of the 13th ICOLD Congress, volume 2, pages 25-34, New Delhi, India, 1979.

Shire, T., O'sullivan, C., and Hanley, K. The influence of fines content and size-ratio on the micro-scale properties of dense bimodal materials. Granular Matter, 18(3): $52,2016$.

Shire, T., O’Sullivan, C., Hanley, K., and Fannin, R. J. Fabric and effective stress distribution in internally unstable soils. Journal of Geotechnical and Geoenvironmental Engineering, 140(12):04014072, 2014.

Skempton, A. and Brogan, J. Experiments on piping in sandy gravels. Geotechnique, 44(3):449-460, 1994.

Sufian, A., Russell, A., and Whittle, A. Anisotropy of contact networks in granular 
media and its influence on mobilised internal friction. Géotechnique, 67(12):10671080, 2017.

Thevanayagam, S. and Mohan, S. Intergranular state variables and stress-strain behaviour of silty sands. Geotechnique, 50(1):1-23, 2000.

Thevanayagam, S., Shenthan, T., Mohan, S., and Liang, J. Undrained fragility of clean sands, silty sands, and sandy silts. Journal of geotechnical and geoenvironmental engineering, 128(10):849-859, 2002.

Vaid, Y. Liquefaction of silty soils. In Prakash, S. and P., D., editors, Ground Failures Under Seismic Conditions, pages 1-16. Geotechnical Special Publication (GSP) 44, 1994.

Vallejo, L. E. Interpretation of the limits in shear strength in binary granular mixtures. Canadian Geotechnical Journal, 38(5):1097-1104, 2001.

Wan, C. F. and Fell, R. Assessing the potential of internal instability and suffusion in embankment dams and their foundations. Journal of geotechnical and geoenvironmental engineering, 134(3):401-407, 2008.

Wichtmann, T., Hernández, M. N., and Triantafyllidis, T. On the influence of a noncohesive fines content on small strain stiffness, modulus degradation and damping of quartz sand. Soil Dynamics and Earthquake Engineering, 69:103-114, 2015.

Xiao, Y., Xiang, J., Liu, H., and Ma, Q. Strength-dilatancy relation of sand containing non-plastic fines. Géotechnique Letters, 7(2):204-210, 2017.

Zhou, W., Wu, W., Ma, G., tat Ng, T., and Chang, X. Undrained behavior of binary granular mixtures with different fines contents. Powder Technology, 340:139 - 153, 2018. 

plastic fines mixtures. Soils and Foundations, 55(1):213-219, 2015. 
Table 1: Data for Gap-Graded Assemblies

\begin{tabular}{|c|c|c|c|c|}
\hline$\chi$ & $f_{c}$ & $N_{p}^{c}$ & $N_{p}^{f}$ & $N_{p}$ \\
\hline \hline 5.71 & 0.18 & 4320 & 64184 & 68504 \\
5.71 & 0.25 & 4232 & 101408 & 105640 \\
5.71 & 0.30 & 4408 & 130072 & 134480 \\
5.71 & 0.35 & 4160 & 150272 & 154432 \\
5.71 & 0.45 & 3488 & 208912 & 212400 \\
\hline 8.00 & 0.18 & 4104 & 155872 & 159976 \\
8.00 & 0.25 & 4744 & 290856 & 295600 \\
7.99 & 0.30 & 4496 & 344784 & 349280 \\
8.00 & 0.35 & 4384 & 398472 & 402856 \\
8.00 & 0.45 & 3376 & 532472 & 535848 \\
\hline 14.24 & 0.18 & 4048 & 910248 & 914296 \\
14.28 & 0.25 & 4056 & 1495848 & 1499904 \\
14.28 & 0.30 & 4440 & 2134240 & 2138680 \\
14.29 & 0.35 & 4184 & 2429848 & 2434032 \\
14.28 & 0.45 & 3328 & 3287256 & 3290584 \\
\hline
\end{tabular}




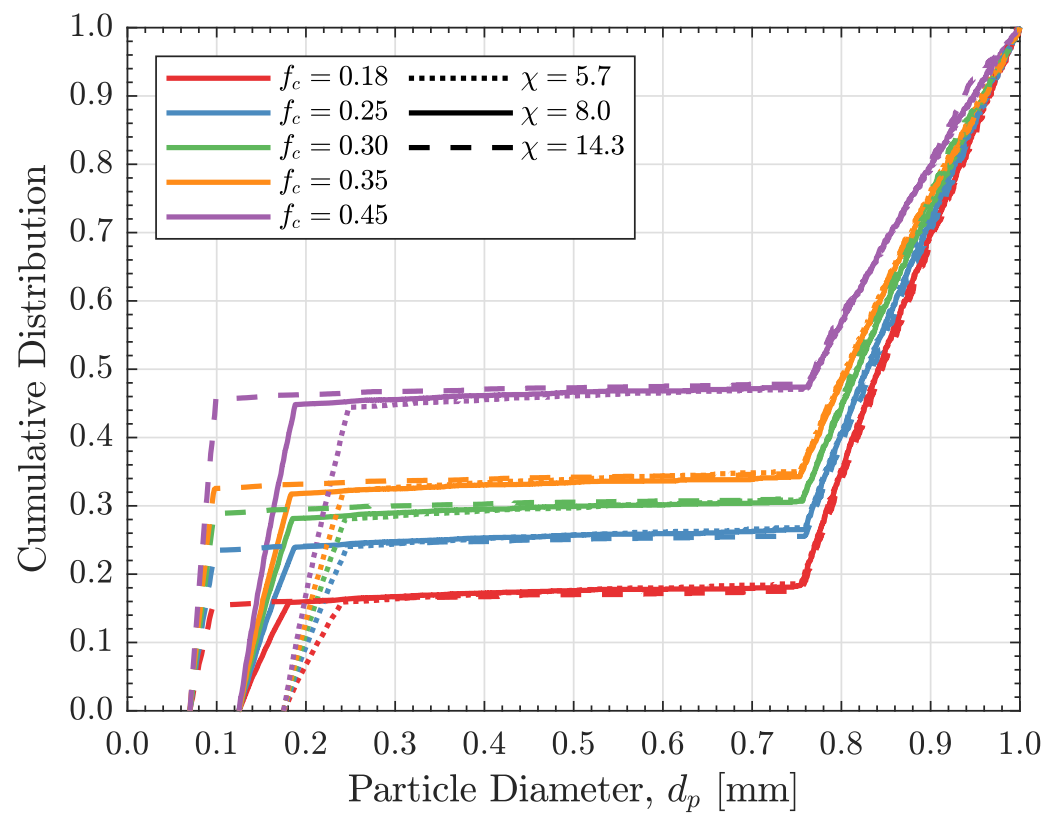

Figure 1: Particle size distribution of the gap-graded assemblies considered in this study. Note the colour scheme differentiating the various fines content, $f_{c}$, which is maintained for the remainder of the paper, unless stated otherwise. 


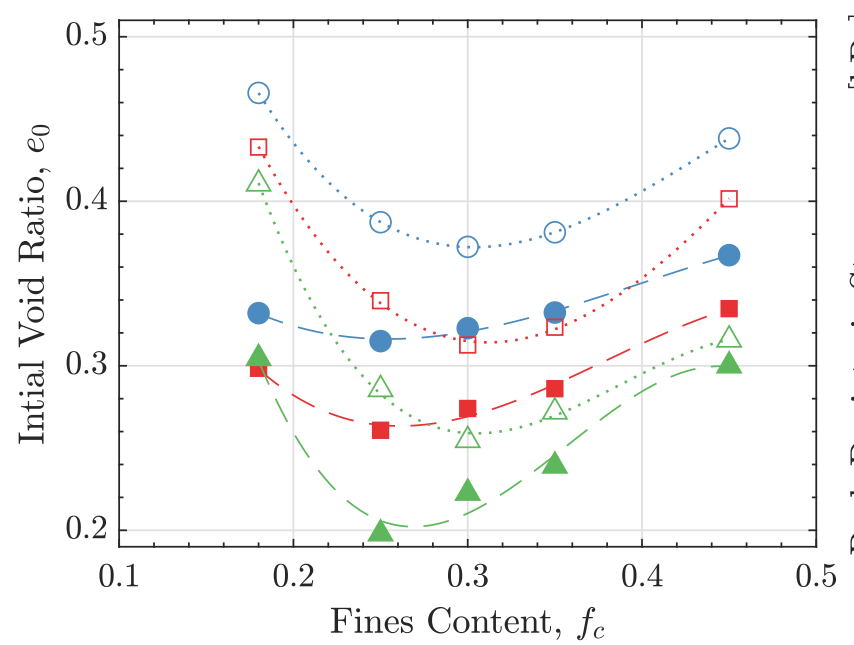

(a)

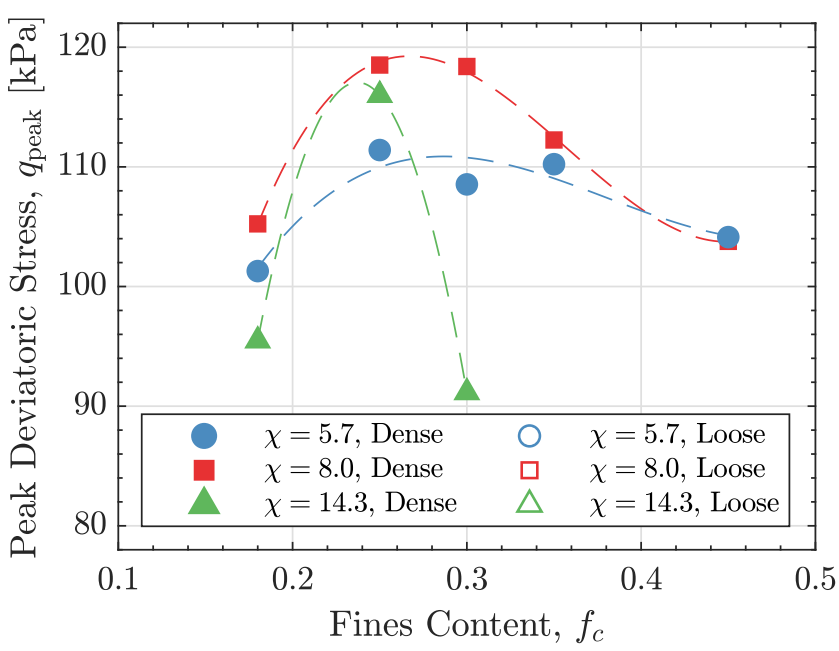

(b)

Figure 2: Initial void ratio, $e_{0}$, and peak deviatoric stress, $q_{\text {peak }}$ at varying fines content and particle size ratio. The dashed/dotted lines are cubic polynomial best-fits of the dense/loose data points for indicative purposes only.

(a) $\chi=5.7$ Dense

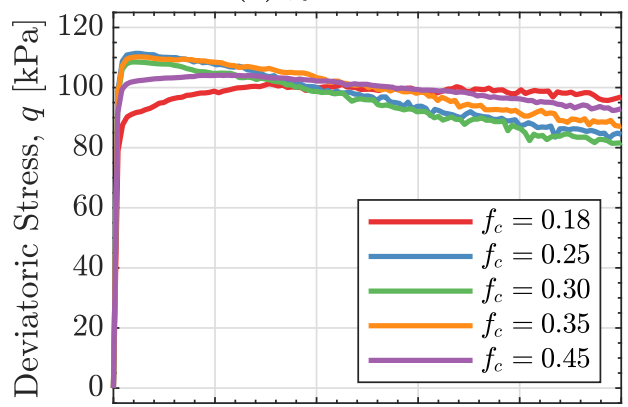

(d) $\chi=5.7$ Loose

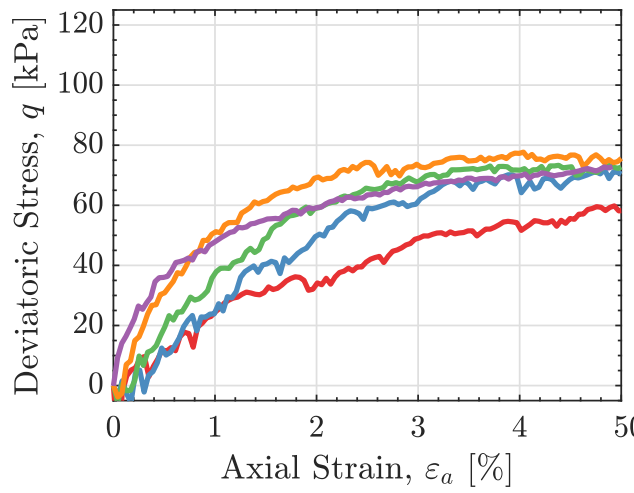

(b) $\chi=8.0$ Dense

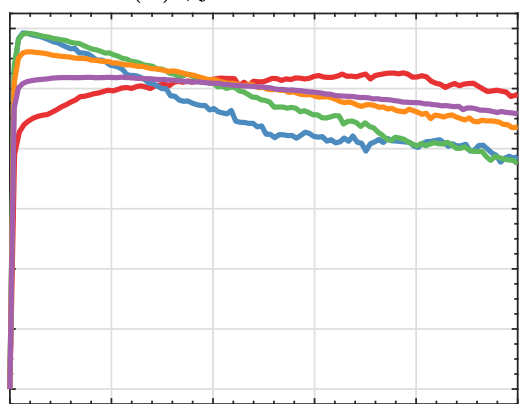

(e) $\chi=8.0$ Loose

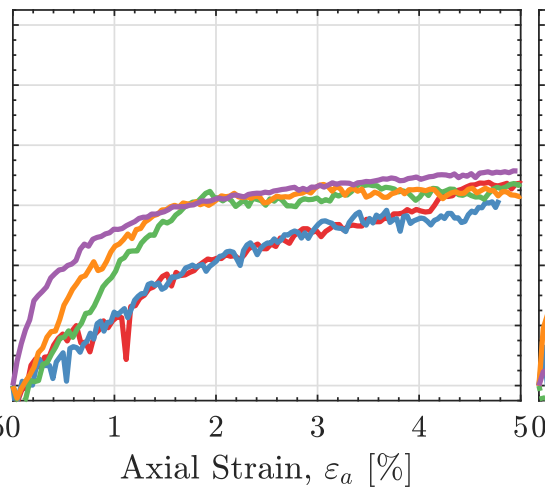

(c) $\chi=14.3$ Dense

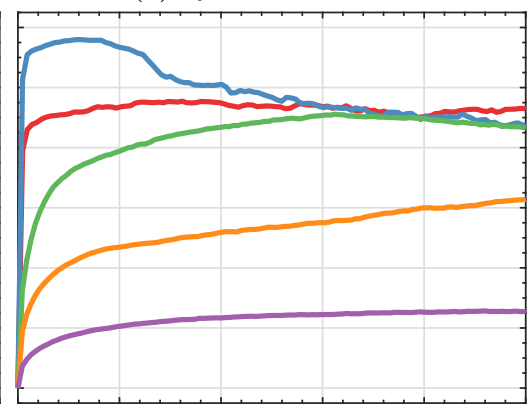

(f) $\chi=14.3$ Loose

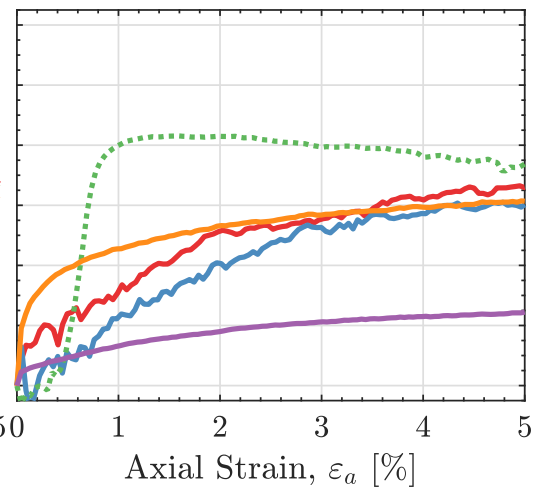

Figure 3: Deviatoric stress during constant mean stress triaxial shearing. Note that the loose case for $\chi=14.3, f_{c}=0.30$ in Fig. $3(\mathrm{f})$ is shown as a dotted line, as this case exhibited an initial metastable response. The dotted line for this case is maintained for all subsequent figures, unless stated otherwise. 


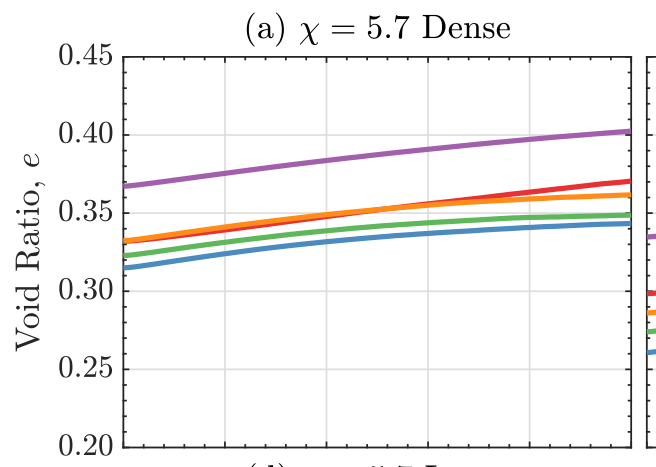

(b) $\chi=8.0$ Dense

(c) $\chi=14.3$ Dense
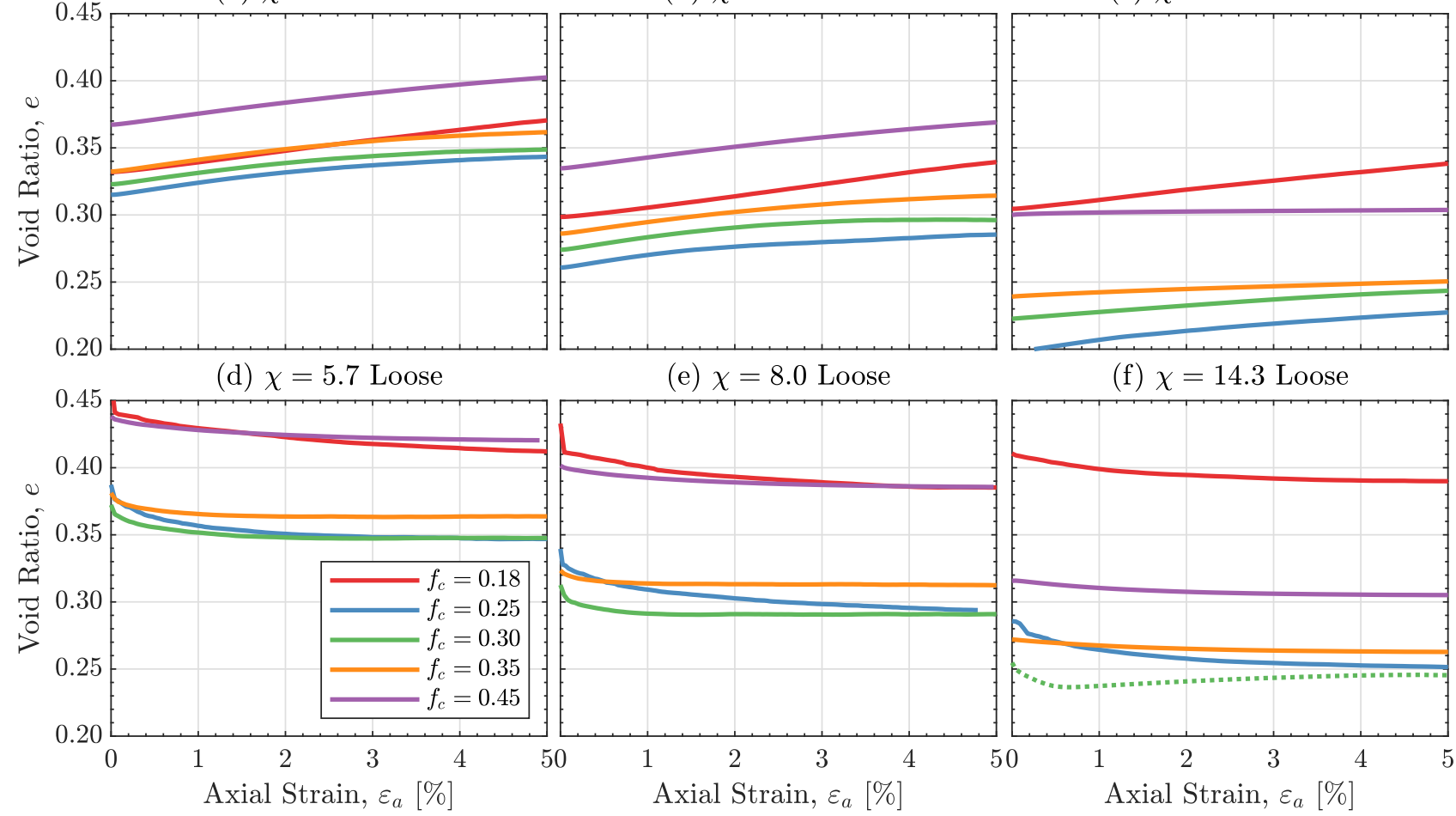

(e) $\chi=8.0$ Loose

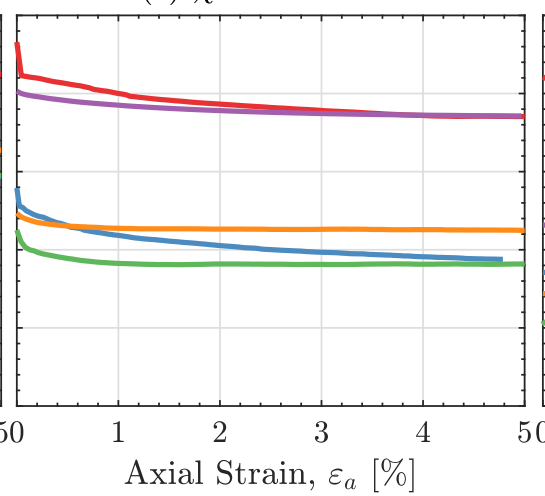

(f) $\chi=14.3$ Loose

Figure 4: Void ratio during constant mean stress triaxial shearing.

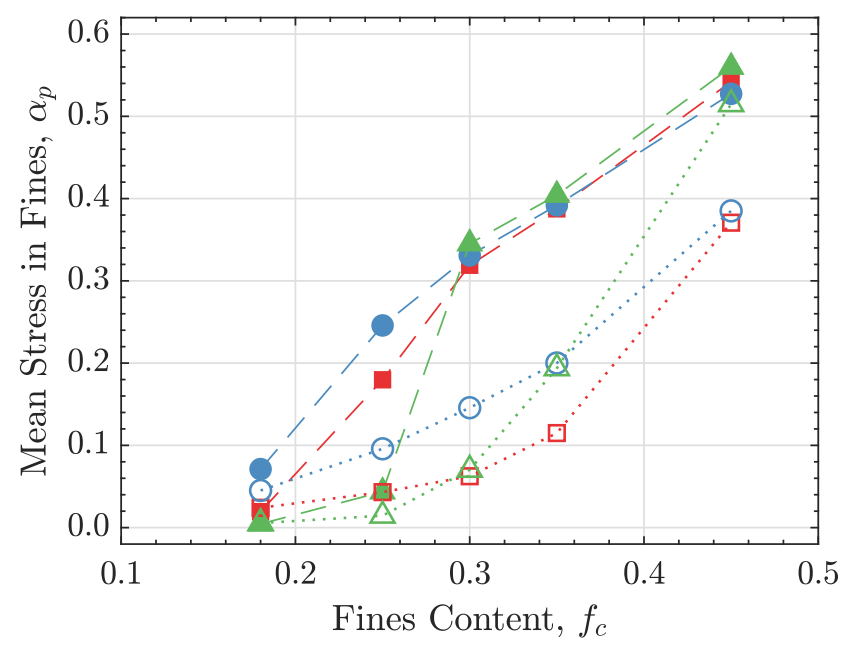

(a) Start of shearing

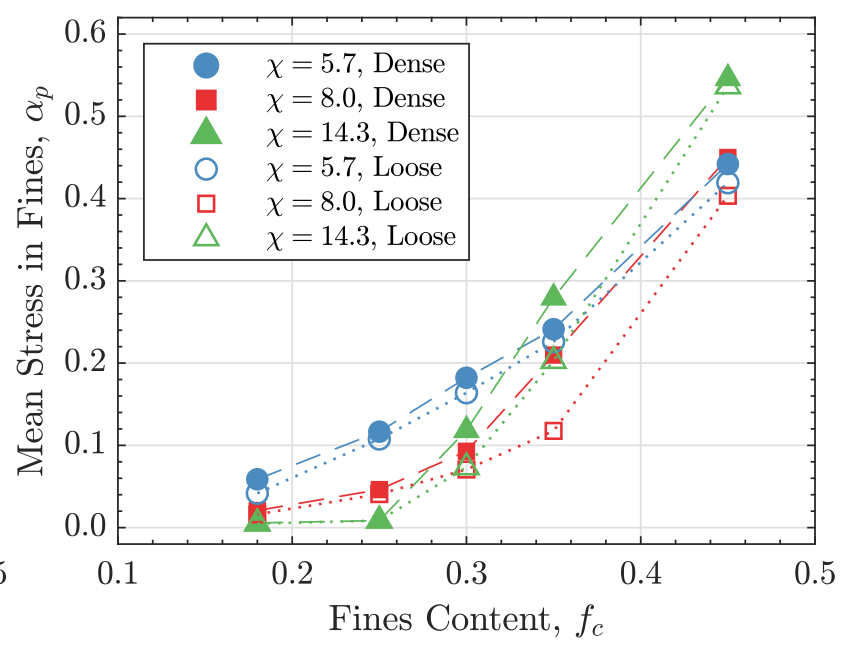

(b) End of shearing

Figure 5: Proportion of mean stress carried by the finer fraction at the start and end of triaxial shearing. 
(a) $\chi=5.7$ Dense

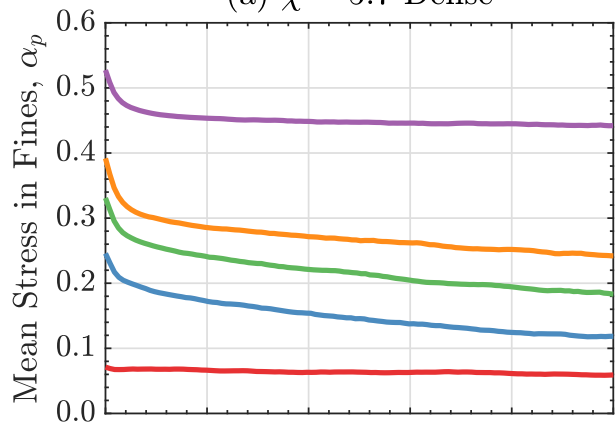

(d) $\chi=5.7$ Loose

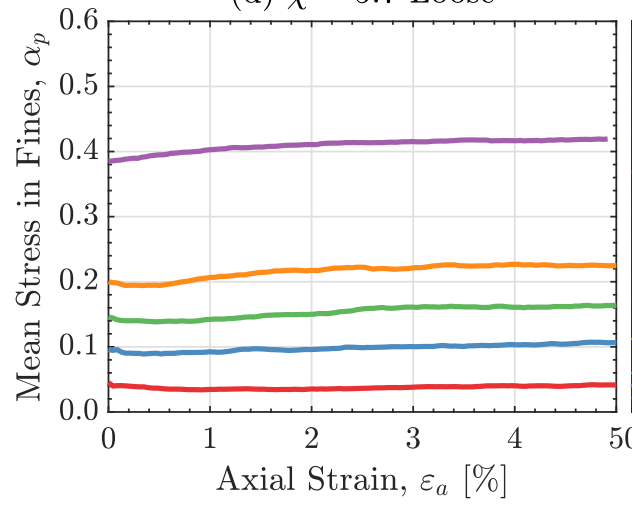

(b) $\chi=8.0$ Dense

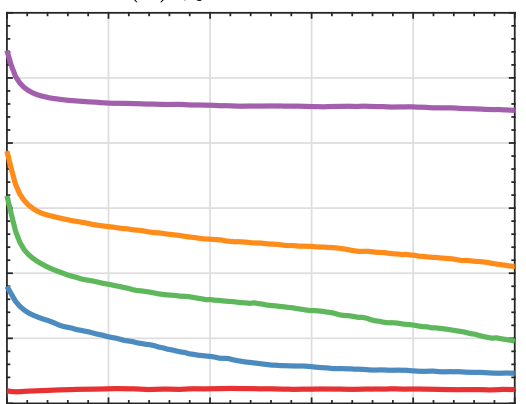

(e) $\chi=8.0$ Loose

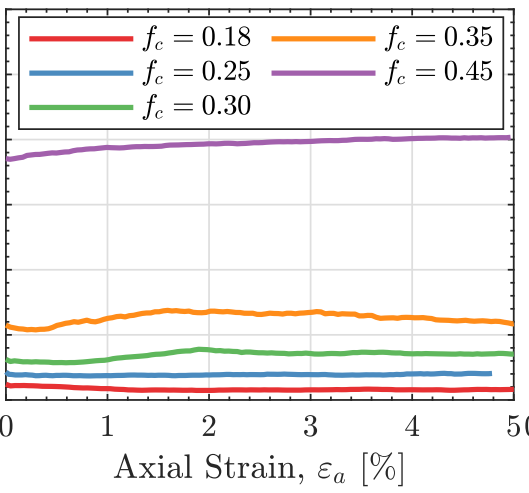

(c) $\chi=14.3$ Dense

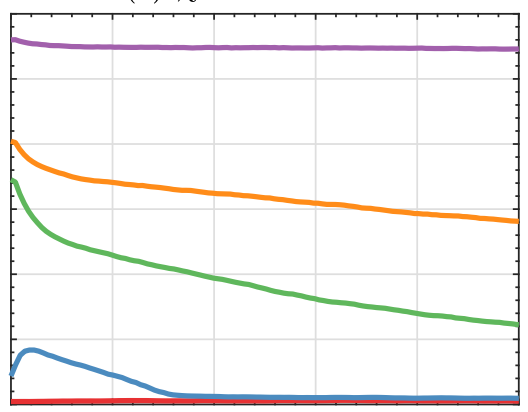

(f) $\chi=14.3$ Loose

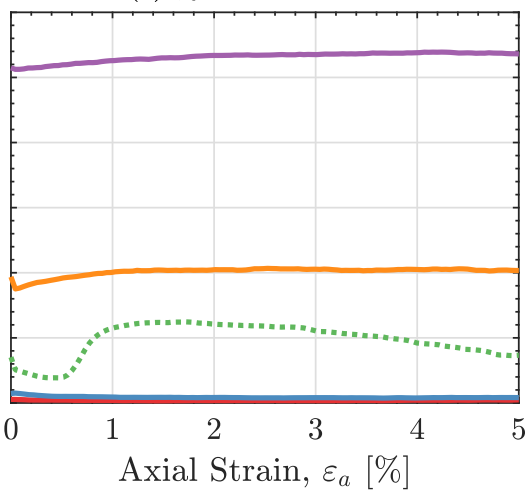

Figure 6: Proportion of mean stress carried by the finer fraction during constant mean stress triaxial shearing. 


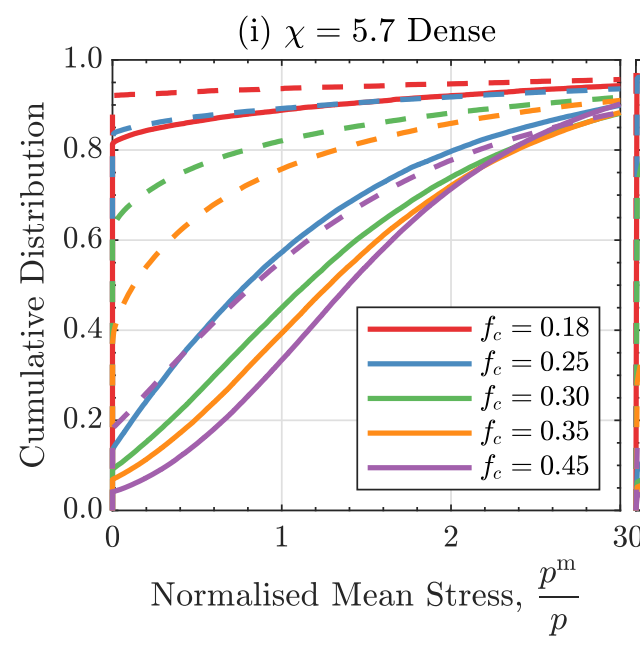

(ii) $\chi=8.0$ Dense

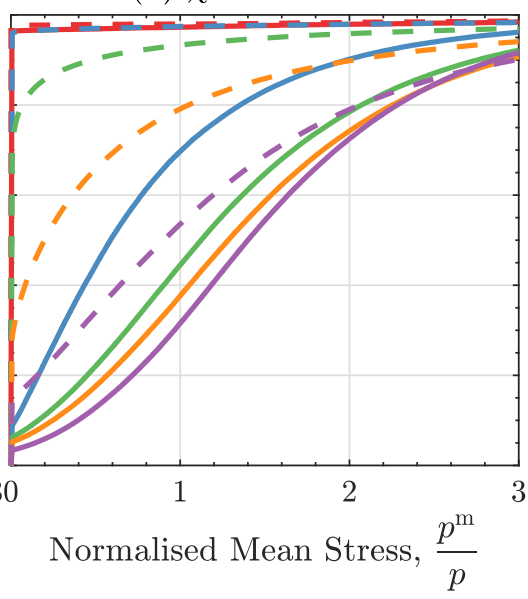

(a) Finer Fraction

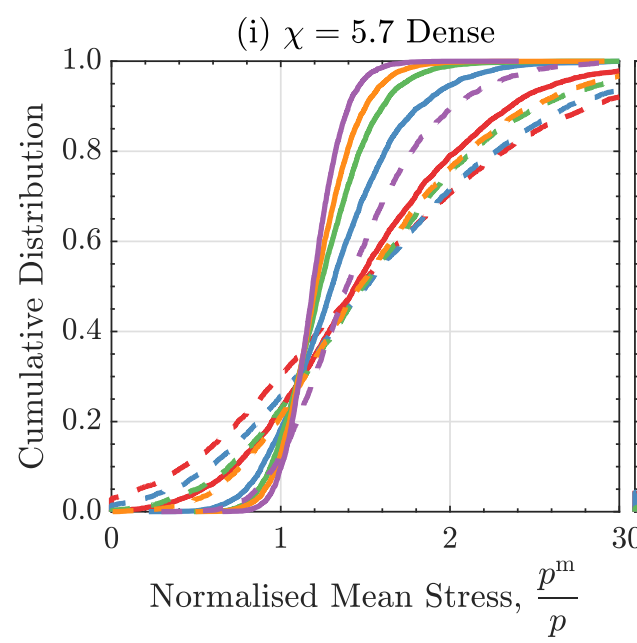

(ii) $\chi=8.0$ Dense

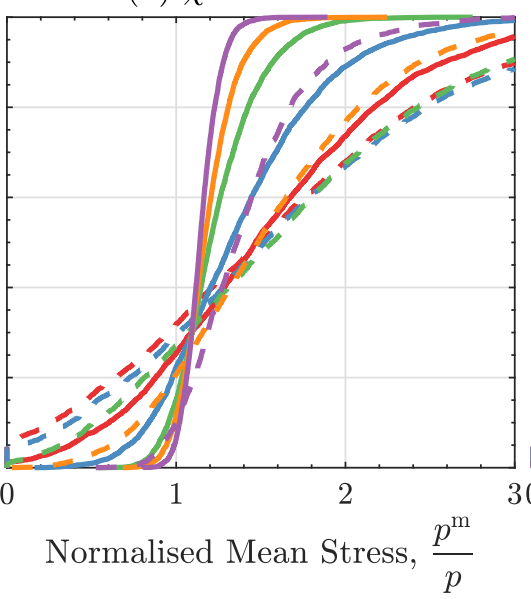

(iii) $\chi=14.3$ Dense

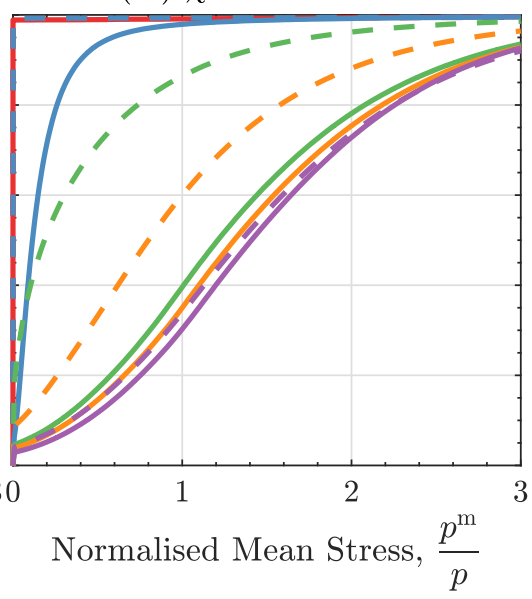

(iii) $\chi=14.3$ Dense

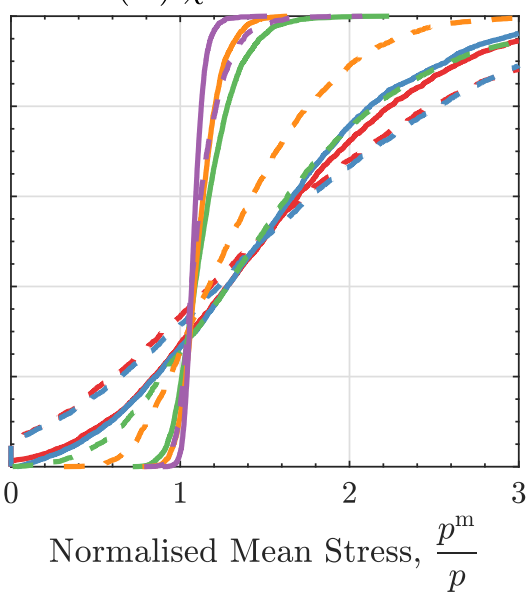

(b) Coarse Fraction

Figure 7: Cumulative distribution by volume of the normalised mean stress on an individual particle. Note that only the results for the dense cases are shown at the start (solid line) and end (dashed line) of shearing. 


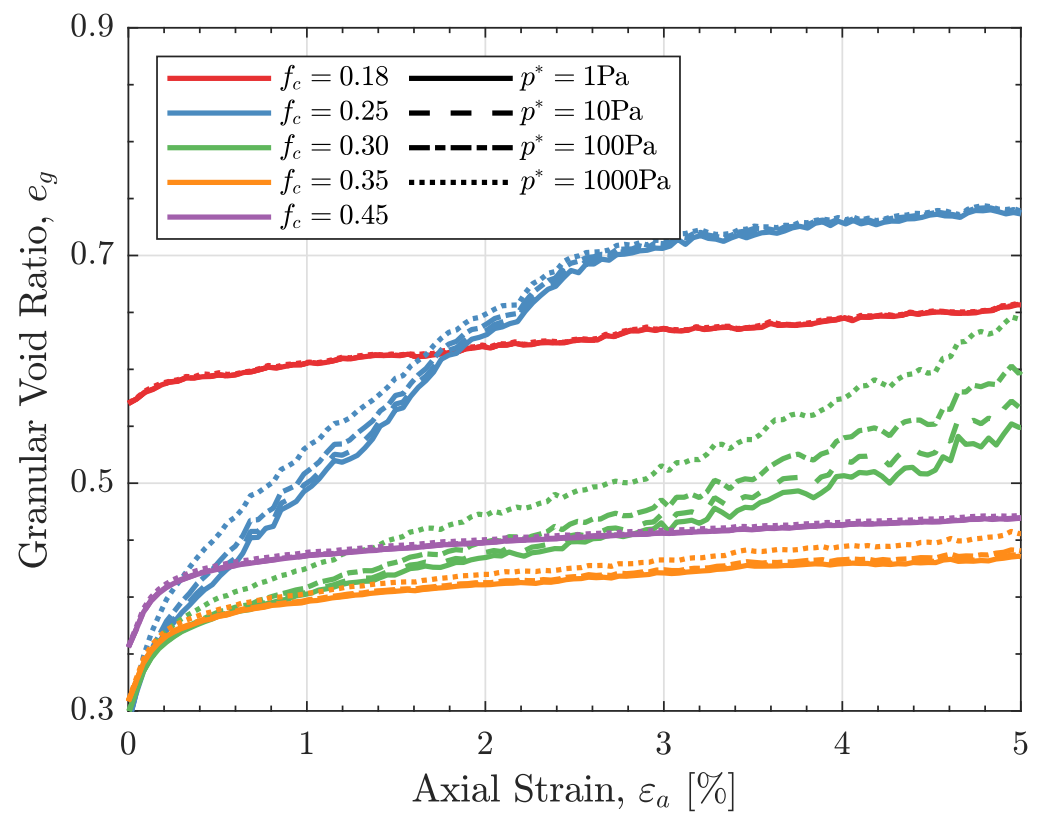

Figure 8: The granular void ratio calculated using different threshold mean stress, $p^{*}$. Note that only the results for the dense $\chi=8.0$ cases are shown.

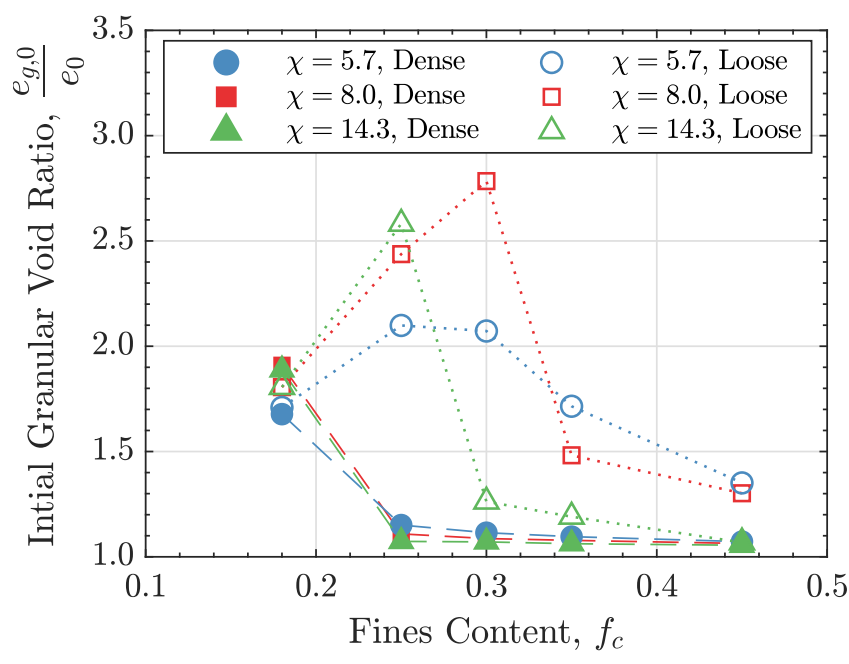

(a)

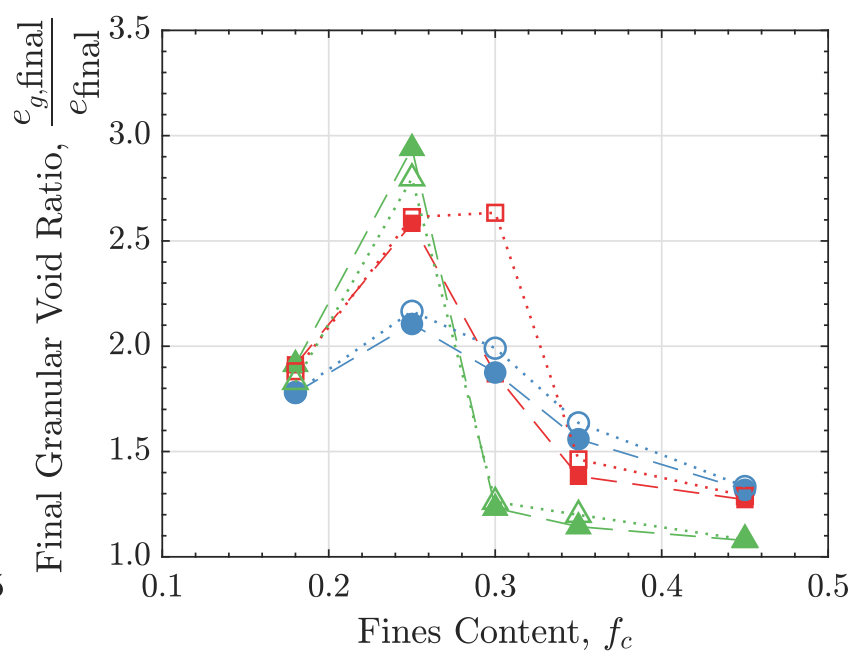

(b)

Figure 9: The granular void ratio at the start $\left(e_{g, 0}\right)$ and end $\left(e_{g, \text { final }}\right)$ of shearing, normalised by the macroscopic void ratio. A threshold mean stress of $p^{*}=1 \mathrm{~Pa}$ is considered for $e_{g}$. 
(a) $\chi=5.7$ Dense

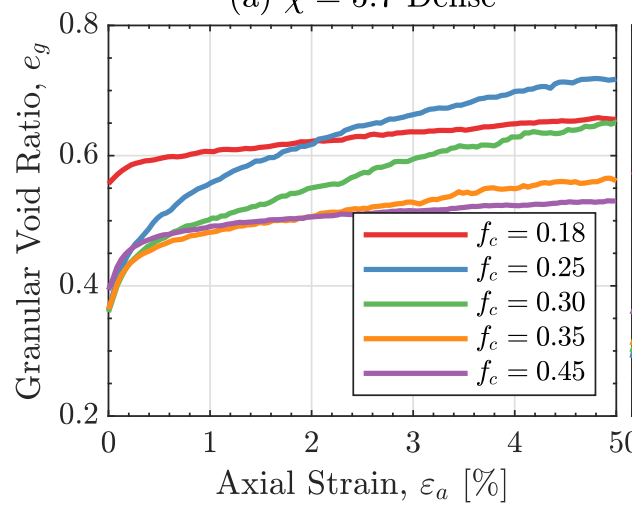

(b) $\chi=8.0$ Dense

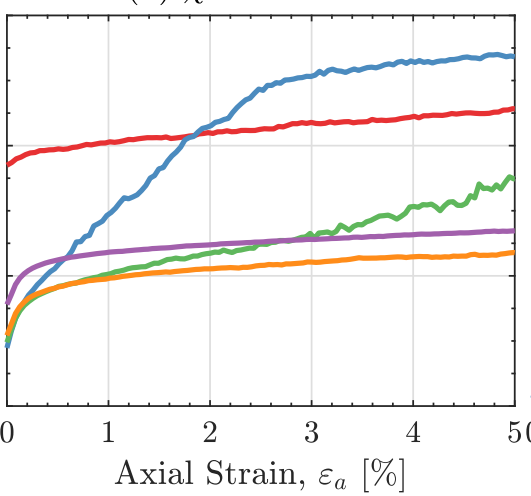

(c) $\chi=14.3$ Dense

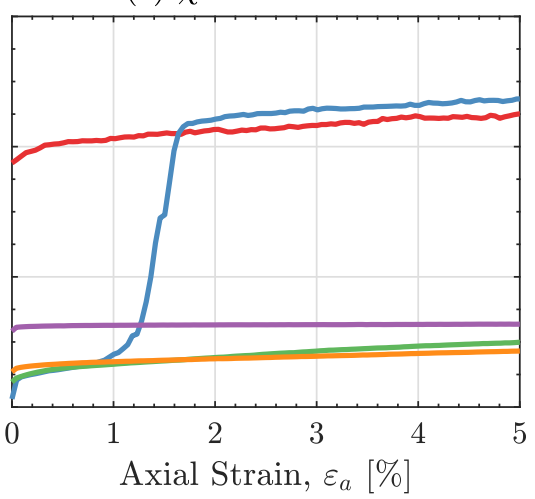

Figure 10: Granular void ratio, $e_{g}$ (Eq. 11), during constant mean stress triaxial shearing. A threshold mean stress of $p^{*}=1 \mathrm{~Pa}$ is considered for $e_{g}$.

(a) $\chi=5.7$ Dense

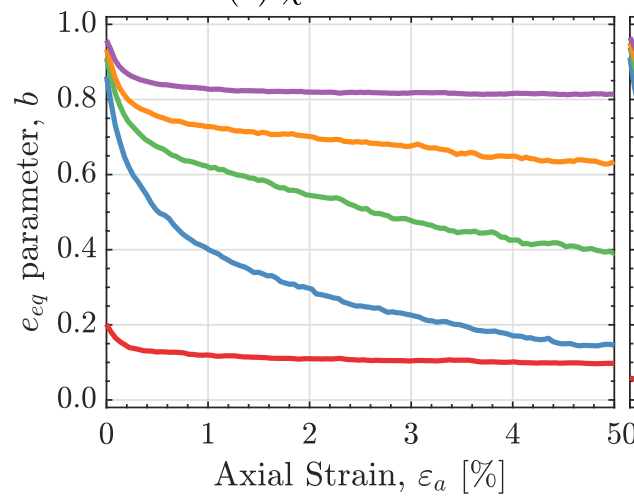

(b) $\chi=8.0$ Dense

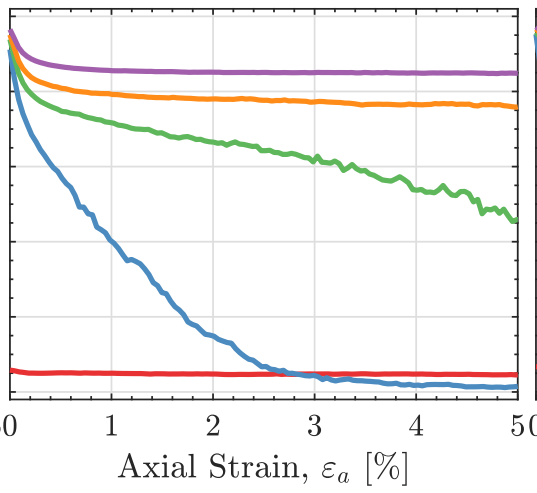

(c) $\chi=14.3$ Dense

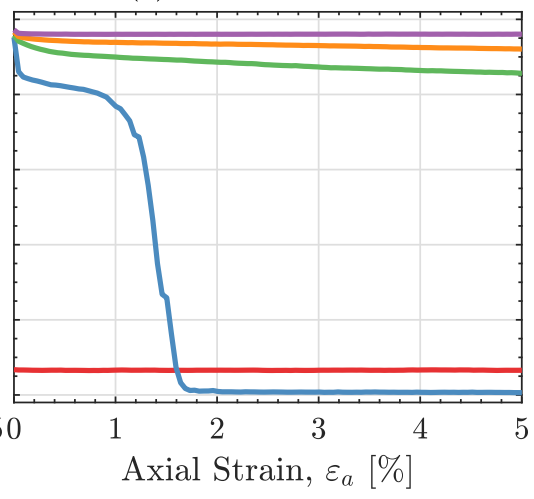

Figure 11: The $b$ parameter in the expression for the equivalent granular void ratio (Eq. 7) is also shown, as calculated using Eq. 9. 
(i) $\chi=5.7$

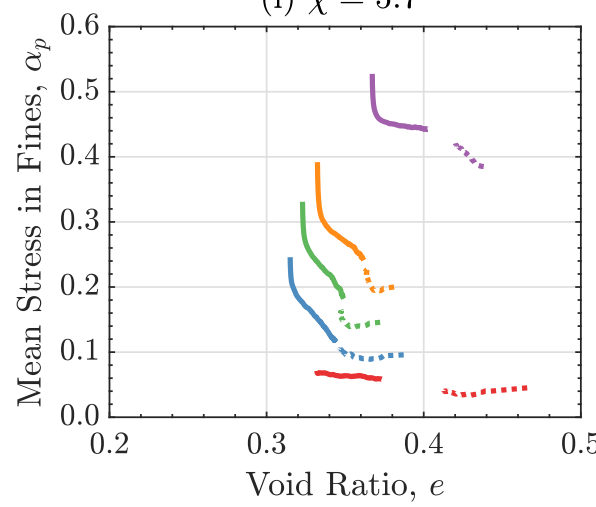

(i) $\chi=5.7$

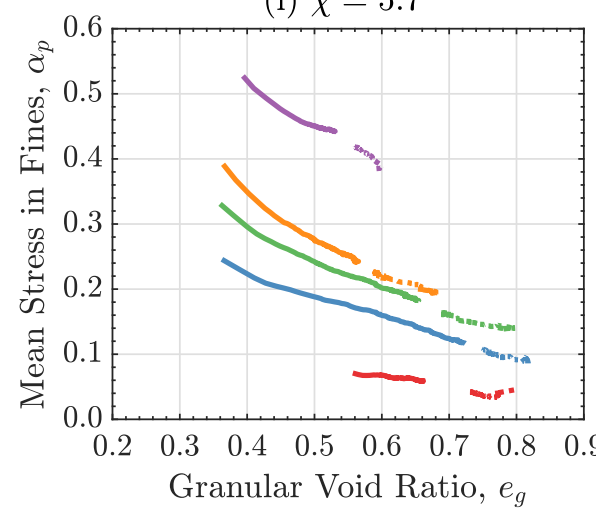

(ii) $\chi=8.0$

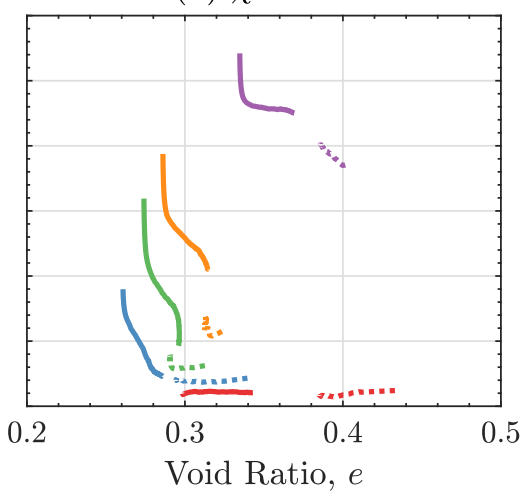

(a)

(ii) $\chi=8.0$

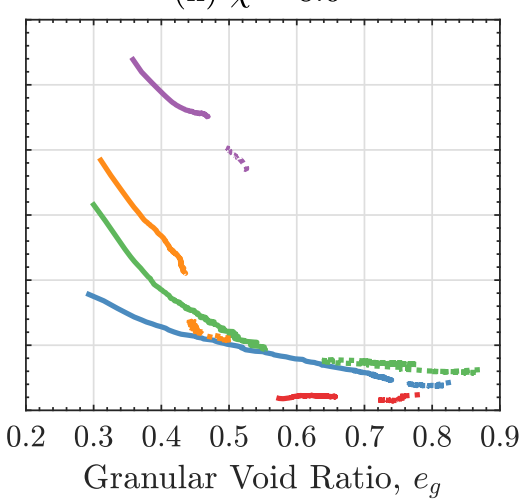

(b) (iii) $\chi=14.3$

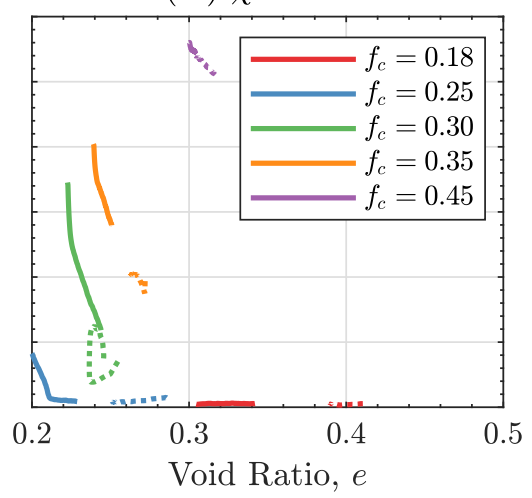

(iii) $\chi=14.3$

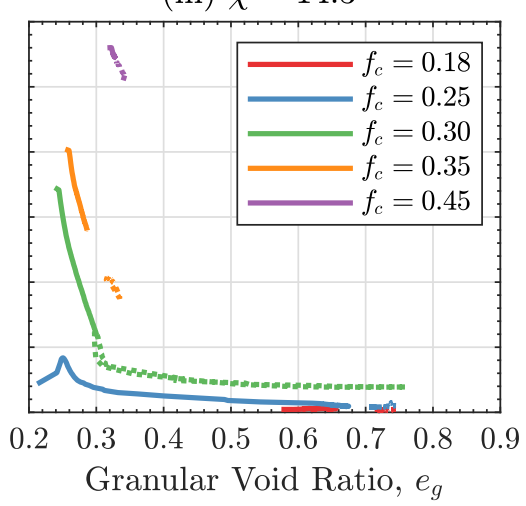

Granular Void Ratio, $e_{g}$

Figure 12: Relationship between $\alpha_{p}$ and the standard definition of void ratio, $e$, in addition to the granular void rati, $e_{g}$. Note that dense samples are shown as solid lines, while loose samples are shown as a dotted line. 


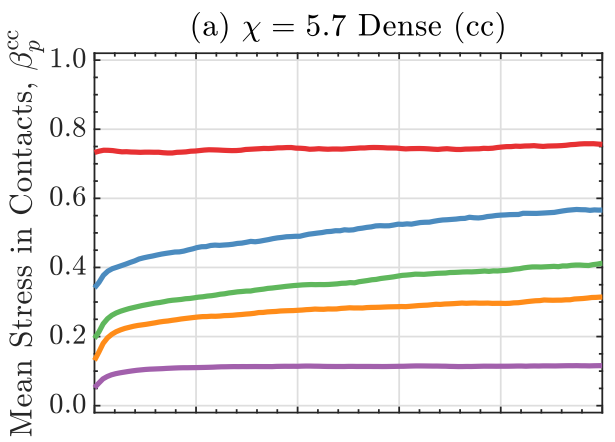

(b) $\chi=8.0$ Dense (cc)

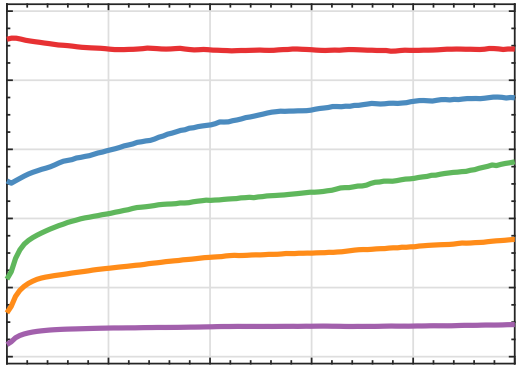

(e) $\chi=8.0$ Dense (fc)

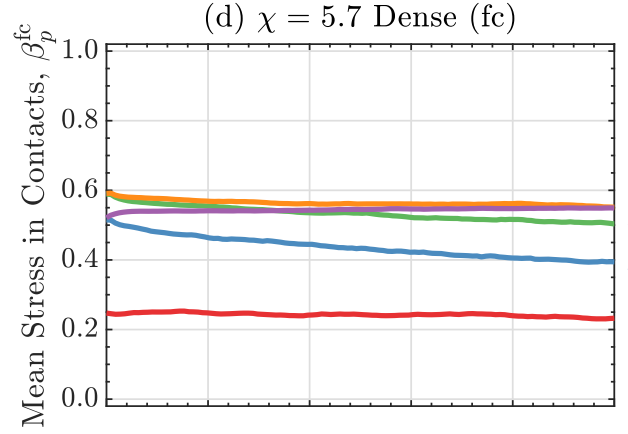

(g) $\chi=5.7$ Dense (ff)

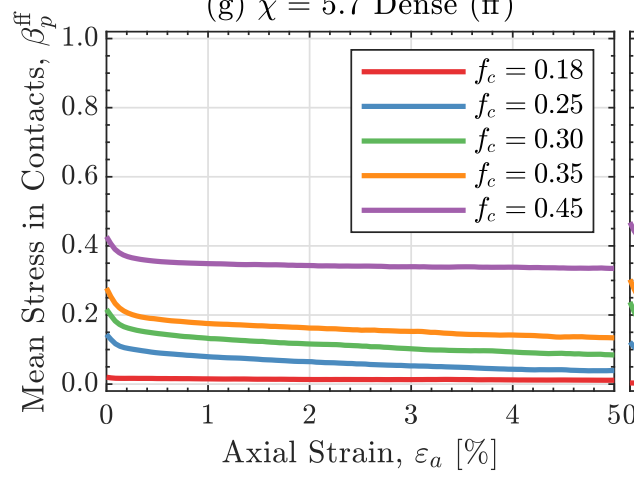

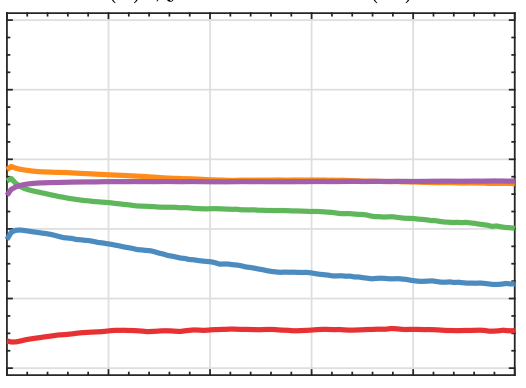

(h) $\chi=8.0$ Dense (ff)

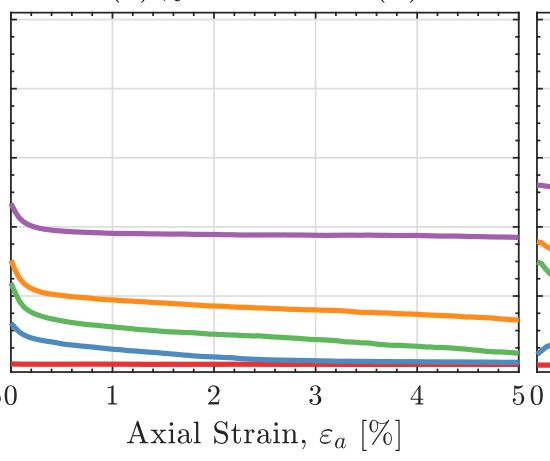

(c) $\chi=14.3$ Dense (cc)

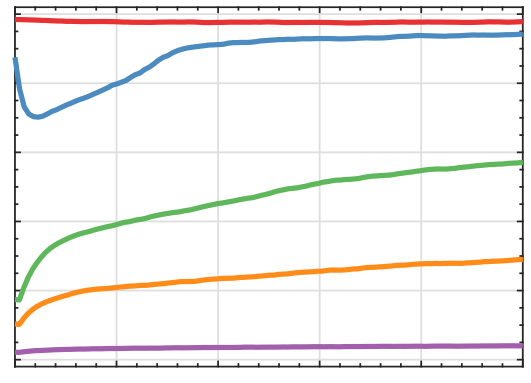

(f) $\chi=14.3$ Dense (fc)

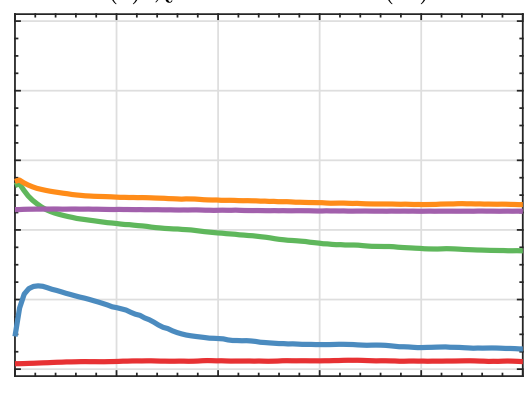

(i) $\chi=14.3$ Dense (ff)

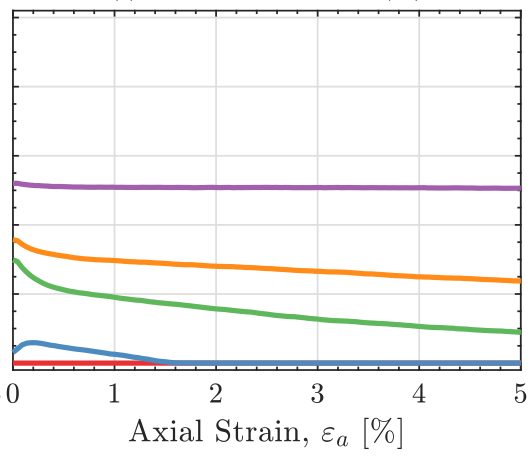

Figure 13: Proportion of mean stress transmitted through the fine-fine (ff), fine-coarse (fc) and coarse-coarse (cc) contacts during constant mean stress triaxial shearing. 


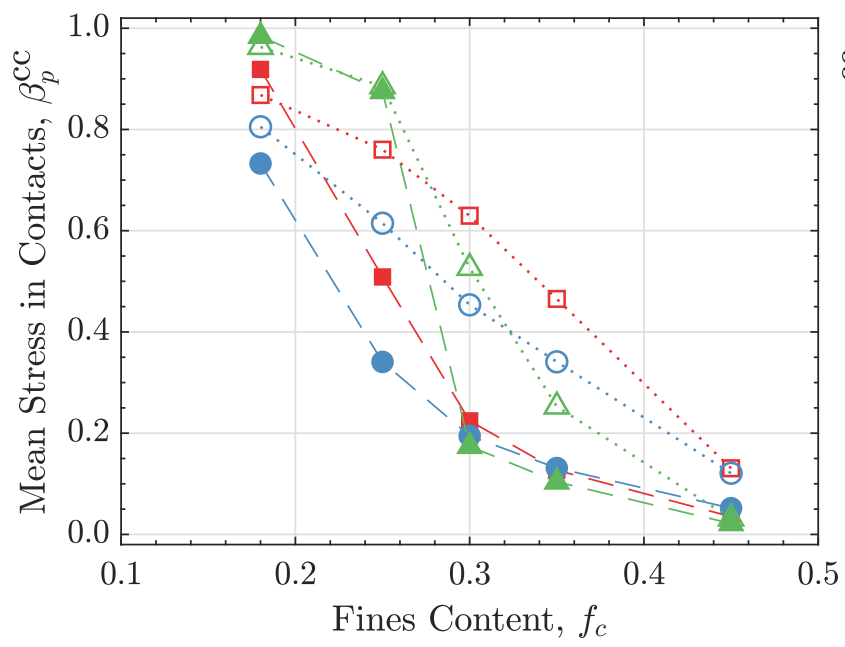

(a) Start of shearing

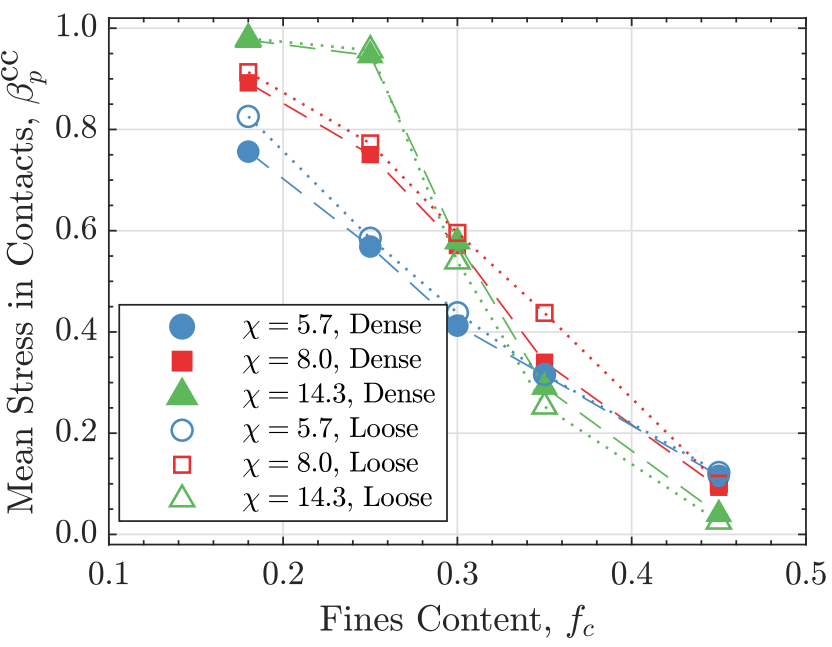

(b) End of shearing

Figure 14: Proportion of mean stress transmitted through the coarse-coarse contacts at the start and end of triaxial shearing.

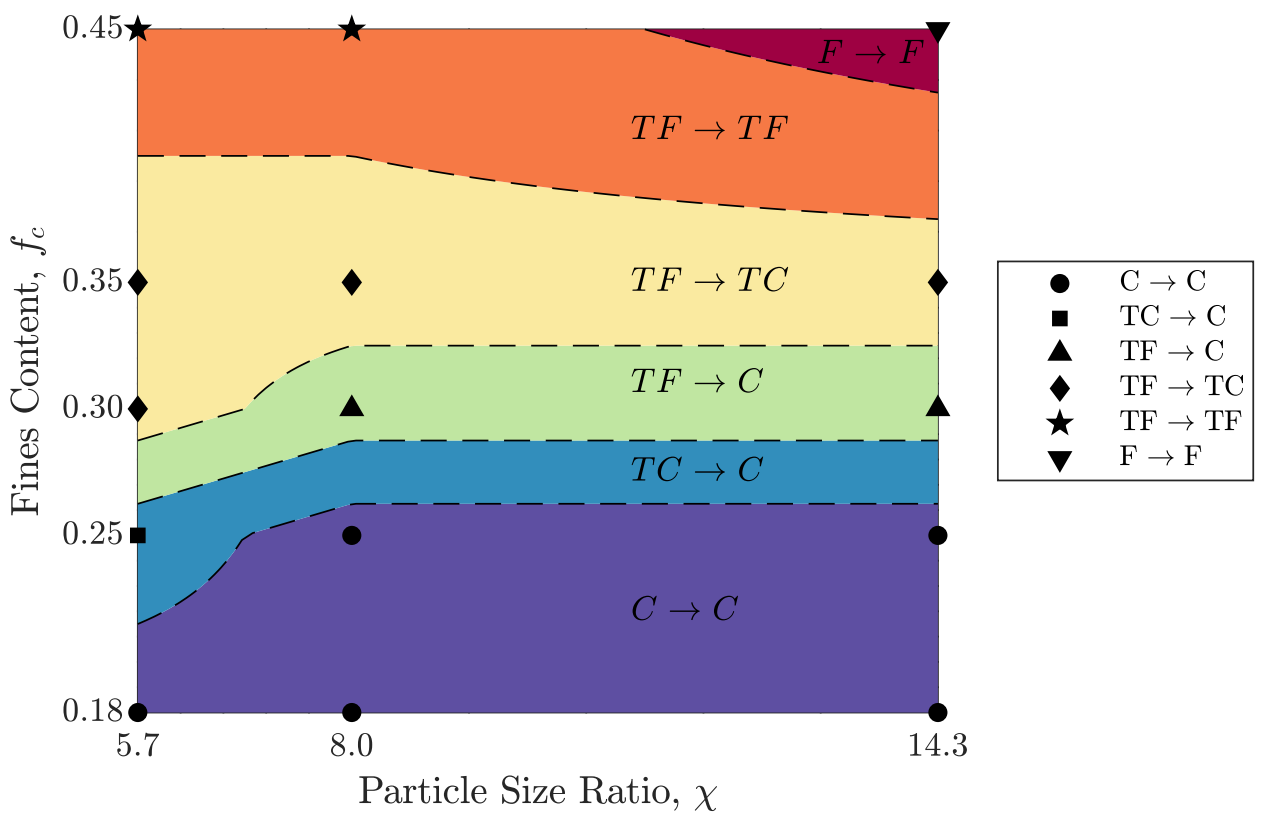

Figure 15: Redistribution of stress in gap-graded assemblies during shearing based on the contact-based stress partition. Note that only dense cases are considered in developing the contour. The contour represents approximate delineation (hence, the dashed lines) based on linear interpolation from the available data points shown by the markers. 


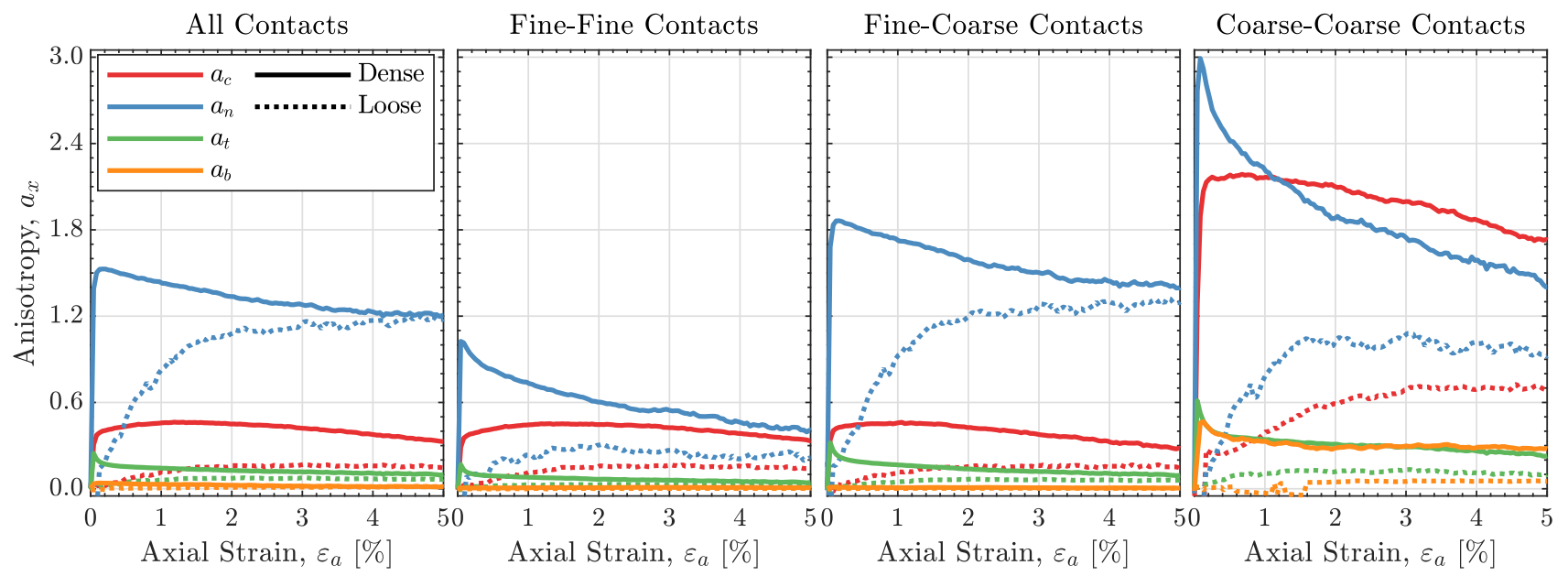

Figure 16: Anisotropy parameters in the partitioned SFF relationship for a typical case $\left(\chi=8.0, f_{c}=0.35\right)$.

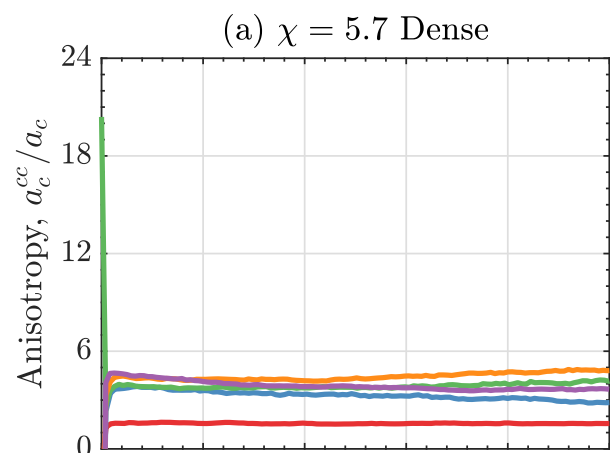

(d) $\chi=5.7$ Dense

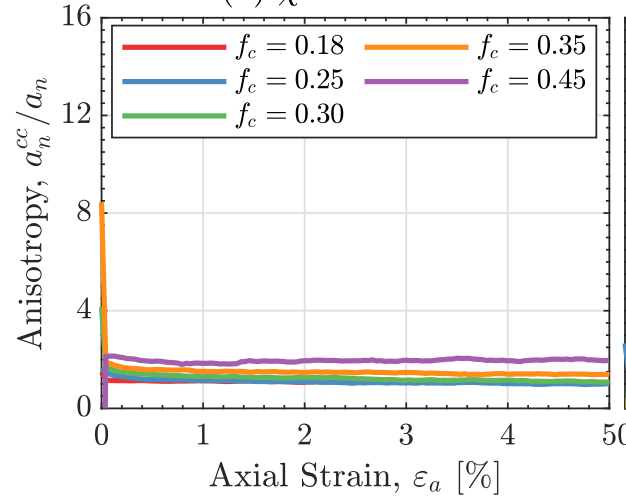

(b) $\chi=8.0$ Dense

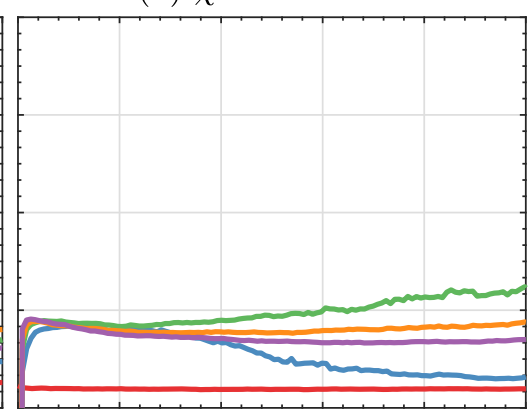

(e) $\chi=8.0$ Dense

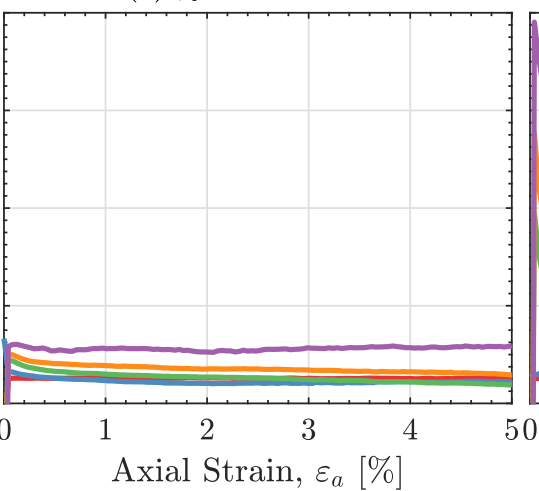

(c) $\chi=14.3$ Dense

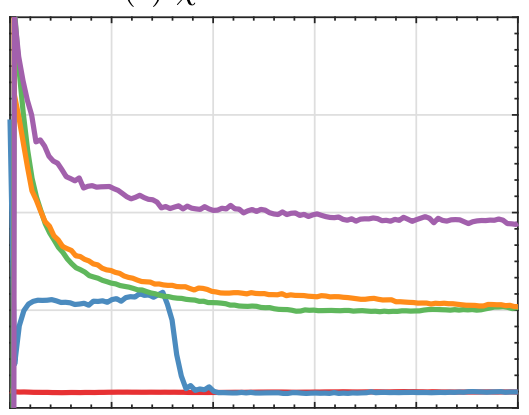

(f) $\chi=14.3$ Dense

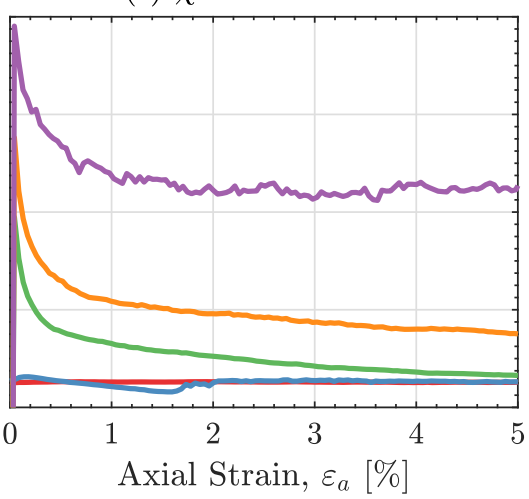

Figure 17: Anisotropy in contact normal orientation $\left(a_{c}^{\mathrm{cc}}\right)$ and normal force magnitude $\left(a_{n}^{\mathrm{cc}}\right)$ for the coarse-coarse contacts (normalised by the anisotropy for all contacts). Note that only the dense cases are shown. 